\title{
Tradeoff Analysis and Joint Optimization of Link-Layer Energy Efficiency and Effective Capacity Toward Green Communications
}

\author{
Wenjuan Yu, Leila Musavian, Member, IEEE, and Qiang Ni, Senior Member, IEEE
}

\begin{abstract}
A joint optimization problem of link-layer energy efficiency (EE) and effective capacity (EC) in a Nakagami- $m$ fading channel under a delay-outage probability constraint and an average transmit power constraint is considered and investigated in this paper. First, a normalized multi-objective optimization problem (MOP) is formulated and transformed into a singleobjective optimization problem (SOP), by applying the weighted sum method. The formulated SOP is then proved to be continuously differentiable and strictly quasiconvex in the optimum average input power, which turns out to be a cup shape curve. Furthermore, the weighted quasiconvex tradeoff problem is solved by first using Charnes-Cooper transformation and then applying Karush-Kuhn-Tucker (KKT) conditions. The proposed optimal power allocation, which includes the optimal strategy for the link-layer EE-maximization problem and the EC-maximization problem as extreme cases, is proved to be sufficient for the Pareto optimal set of the original EE-EC MOP. Moreover, we prove that the optimum average power level monotonically decreases with the importance weight, but strictly increases with the normalization factor, the circuit power and the power amplifier efficiency. Simulation results confirm the analytical derivations and further show the effects of fading severeness and transmission power limit on the tradeoff performance.
\end{abstract}

Index Terms-Quality-of-service, delay-outage probability constraint, effective capacity, energy efficiency, multi-objective optimization problem, weighted sum method.

\section{INTRODUCTION}

W IRELESS communication sector is the fastest growing segment of the communications industry [1]. According to International Telecommunication Union, the number of mobile subscriptions worldwide is approaching the number of people on the earth [2]. In addition, many new wireless applications, such as autonomous driving, smart cities, smart homes and appliances have emerged from research ideas to concrete systems [3]. The explosive growth of wireless communication applications coupled with the proliferation of

Manuscript received April 25, 2015; revised September 22, 2015 and December 21, 2015; accepted January 3, 2016. Date of publication January 21, 2016; date of current version May 6, 2016. This work was supported in part by the China Scholarship Council, UK EPSRC under Grant EP/K011693/1 and the EU FP7 under Grant PIRSES-GA-2013-610524. Part of this work was presented at the IEEE ICC, London, U.K., June 2015. The associate editor coordinating the review of this paper and approving it for publication was N. Prasad.

The authors are with the School of Computing and Communications, InfoLab21, Lancaster University, Lancaster LA1 4WA, U.K. (e-mail: w.yu1@ lancaster.ac.uk, 1.musavian@lancaster.ac.uk, q.ni@lancaster.ac.uk).

Color versions of one or more of the figures in this paper are available online at http://ieeexplore.ieee.org.

Digital Object Identifier 10.1109/TWC.2016.2520472 mobile devices has dramatically speeded up the progress of wireless networks, which results in a higher-quality human life and rapid economic growth. Meanwhile, many technical challenges still remain unsolved in wireless network designs, e.g., the need for reducing energy consumption and end-to-end latency [3].

According to [4], for every 1 TeraWatt hour (TWh) energy consumption, the information and communication technology (ICT) sector is responsible for approximately 0.75 million tons of $\mathrm{CO}_{2}$ gas emissions. If no action is taken, the overall costs and risks of climate change, as a result of the increasing green house gases (GHG) emissions, will be equivalent to losing at least $5 \%$ of global gross domestic product (GDP) every year [5]. Nevertheless, it is also well known that ICT industry has the potential to reduce more than $23 \%$ of its current GHG emissions [5]. Interestingly, if one-third of the GHG emissions is reduced, the generated economical benefit will be higher than the required investment [6]. As an important part of ICT, wireless communication sector needs to take the responsibility to save more energy. Green communication technology, which emphasizes energy efficiency (EE) in addition to spectral efficiency (SE), has thereby been proposed as an effective solution which not only benefits communication technology sector, but also promotes economic and ecological sustainability. However, considering the compromise between network performance and energy savings, designing an efficient resource allocation strategy to limit the network energy consumption is a real challenge [7]-[9].

In this trend, an energy-efficient optimization problem to maximize the worst-case link EE was formulated and studied in [10], under the rate, transmit power, and subcarrier assignment constraints. Price-driven algorithms for joint power and admission control are proposed to characterize the tradeoff between the total energy consumption and the system capacity in [11]. EE and SE tradeoff, based on Shannon limit, has also been extensively studied for different kinds of wireless communication networks, such as energy-constrained wireless multi-hop networks with a single source-destination pair [12], multi-user downlink orthogonal frequency division multiple access (OFDMA) networks [13], general narrowband interference-limited systems [14] and OFDMA-based cooperative cognitive radio networks [15]. The relationship between EE and SE for downlink multiuser distributed antenna systems with proportional fairness was investigated in [16]. Specifically, the EE-maximization problem was first converted into a 
multi-objective optimization problem (MOP), by maximizing the numerator of EE while minimizing its denominator. Then, the MOP was transformed into a single-objective optimization problem (SOP) using weighted sum method, and the optimal power value was provided by applying Lagrangian method and sub-gradient iteration approach. Considering imperfect channel estimation in an orthogonal frequency division multiplexing (OFDM) network, the inverse of EE and inverse of SE were combined into a weighted optimization problem in [17]. The problem was then transformed into a convex problem, namely, to jointly minimize the total power consumption and maximize the channel capacity, which was solved using Lagrangian method.

In the aforementioned studies [12]-[17], Shannon limit was utilized as the system throughput, which is mostly considered as the suitable capacity metric for communication systems with no link-layer delay quality-of-service (QoS) requirements. Nevertheless, for delay-sensitive mobile multimedia applications, such as video conferencing, autonomous driving and online gaming, provisioning QoS requirements is critical. Actually, 5G, the next generation of mobile communication technology, has been anticipated to not only offer $>1$ Gbps downlink data rate, but also sub-1ms end-to-end latency and $90 \%$ reduction in network energy usage [3]. Henceforth, it is important and imperative to investigate EE and SE based on a link-layer wireless channel model, which explicitly characterizes the channel in terms of link-layer QoS metrics [18].

Effective capacity (EC), as a generalized link-level capacity notion which specifies the maximum arrival rate with a target delay-outage probability requirement, has recently received a lot of attention [18]. Specifically, EC, can be regarded as the link-layer SE while the link-layer EE can be formulated as the ratio of $\mathrm{EC}$ to the total power expenditure. However, just like the inconsistent property of EE and SE in physical-layer channel model, the link-layer EE and EC also can be incompatible [19]. In more details, for a point-to-point communication system operating in a flat-fading channel, the EE versus EC curve is bell shape when non-zero circuit power is considered [20]. Indeed, the link-layer EE and SE experience a much more pronounced tradeoff, compared to the physical-layer EE and SE [20]-[22]. Therefore, how to allocate system resources to efficiently balance the two conflicting metrics deserves elaborate study. Towards this direction, considering frequency flat-fading channels, an optimal power allocation strategy to maximize EC subject to an EE constraint, for delay-limited mobile multimedia applications was introduced in [21]. [22] analyzed the tradeoff between EE and EC by providing the mutually beneficial $(\mathrm{MB})$ region and the contention-based $(\mathrm{CB})$ region. In more details, the $\mathrm{MB}$ region refers to the case when $\mathrm{EE}$ and $\mathrm{EC}$ can mutually optimize, whereas in the $\mathrm{CB}$ region, the trends of $\mathrm{EE}$ and $\mathrm{EC}$ conflict. However, the adjustable tradeoff between EE and EC, as well as a closed-form power allocation strategy was not involved in [22]. On the other hand, the EE-EC relationship was exploited and plotted, by expressing signal-to-noise ratio (SNR) in terms of SE using a curve fitting method in [23]. We note that, according to users' diverse preferences, various application types and dynamic surrounding circumstances, a more flexible and tractable tradeoff function is preferable, which is not provided in [20]-[23].

In this paper, we consider and investigate a joint maximization problem of link-layer EE and EC under an average input power constraint. Especially, the link-layer EE-EC tradeoff problem includes the physical-layer EE-SE tradeoff problem, zero-outage capacity situation, link-layer EE-maximization problem and link-layer EC-maximization problem, as special cases. We analyze and discuss all these situations and provide simulation results to compare the physical-layer and the link-layer tradeoff performance. For the formulated powerunconstrained EE-EC tradeoff problem, a closed-form expression for the power allocation strategy is first derived to pave the way for the power-constrained problem. Then, we analyze the link-layer EE-EC tradeoff problem under an input power constraint and provide the Pseudocode of the optimal power allocation algorithm in Table I. In order to obtain more insight, we analyze the influence of different system parameters, such as the importance weight, normalization factor, circuit power, power amplifier efficiency and the fading parameter.

In more details, this paper has the following contributions:

- A generalized link-layer EE-EC MOP in a Nakagami-m fading channel under a delay-outage probability constraint and an average transmit power constraint is transformed into an SOP using weighted sum method. Especially, we introduce two normalization values to balance the different measurements and orders of magnitude of EE and EC.

- We prove that the unconstrained EE-EC tradeoff formulation is continuously differentiable, strictly quasiconvex in the average power and follows a cup shape curve. Henceforth, the global optimum is unique and can be achieved at a finite value.

- By using the Charnes-Cooper transformation and KKT conditions, the optimum power allocation scheme for the power-unconstrained link-layer EE-EC tradeoff problem is derived, and is proved to be sufficient for the Pareto optimal set of the original EE-EC MOP. For the powerconstrained tradeoff problem, an optimal power allocation algorithm is provided in Table I.

- We prove that the average optimal power level monotonically decreases with the importance weight, but strictly increases with the normalization factor, circuit power and power amplifier efficiency.

- We finally provide a proper guideline on how to choose the normalization factor and importance weight to benefit either link-layer EE or EC.

The remainder of this paper is organized as follows. In Section II, the system model and a general tradeoff problem formulation are provided. The theory of link-layer EC and EE is introduced in Section III. In Section IV, the optimal power allocation strategy is derived and analyzed. The effects of importance weight, normalization factor, circuit power, and power amplifier efficiency on the average power level are further investigated in this section. Finally, numerical results are given in Section $\mathrm{V}$, followed by conclusions in Section VI. 


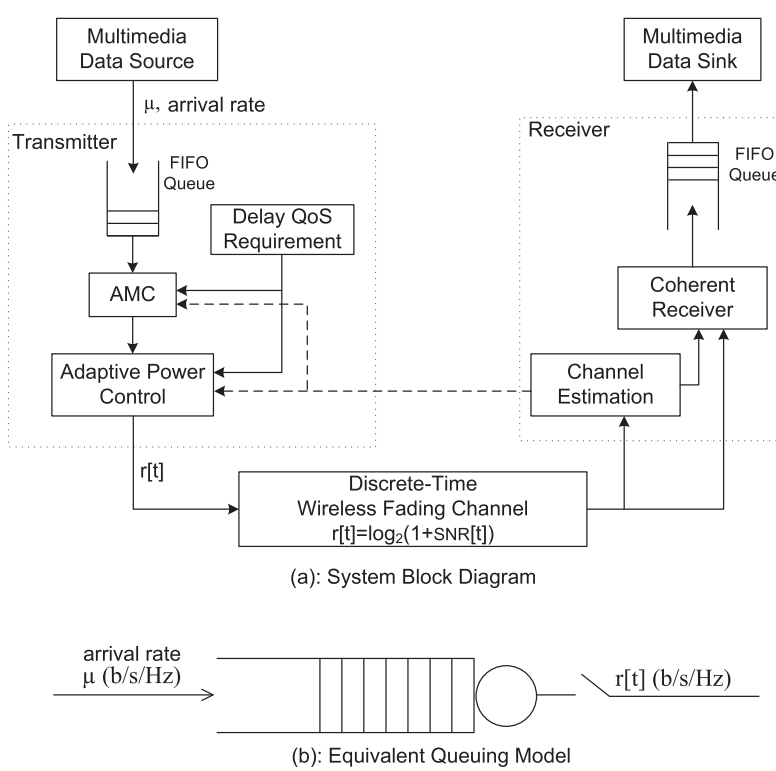

Fig. 1. System model.

\section{System Model AND Problem Formulation}

\section{A. System Model}

A point-to-point wireless communication link over a Nakagami- $m$ flat-fading channel is considered in this paper. Different from the physical-layer channel model which has limitations in QoS support, the link-layer model depicted in Fig 1(a) captures a generalized link-level capacity notion of the fading channel, under a delay QoS requirement [18], [24]. Firstly, the upper-layer packets are divided into frames at the data-link layer. Then, the source traffic and the network service are matched using a first-in-first-out (FIFO) buffer, which prevents loss of packets that could occur when the source rate is higher than the service rate, at the expense of increasing the delay [18]. At the physical layer, the frames stored at the buffer are split into bit streams. Adaptive coding and power allocation strategy are applied at the transmitter [25], using the channel-state information (CSI) fed back from the receiver, and the predetermined delay QoS requirement. The bit streams are read out of the FIFO buffer and transmitted through the wireless fading channel. Finally, the reverse operations are performed at the receiver and the frames are recovered for further processing.

We assume that the wireless channel is block fading, i.e., the channel gain is invariant during each fading-block, but independently varies from one fading-block to another. The length of each fading-block, denoted by $T_{\mathrm{f}}$, is assumed to be an integer multiple of the symbol duration $T_{\mathrm{S}}$. Ideal Nyquist transmission symbol rate is also assumed to be satisfied, which means that the symbol duration $T_{\mathrm{S}}=\frac{1}{B}$, where $B$ is the system bandwidth. In addition, the service rate process, $\{R[t], t=$ $1,2, \ldots\}$, using adaptive transmission is considered to be stationary and ergodic [24]. The instantaneous service rate, in $\mathrm{b} / \mathrm{s} / \mathrm{Hz}$, at the $t^{\text {th }}$ fading-block is given by

$$
R[t]=\log _{2}\left(1+P_{\mathrm{t}}[t] \frac{\gamma[t]}{P_{\mathcal{L}} \sigma_{\mathrm{n}}^{2}}\right),
$$

where $P_{\mathrm{t}}[t]$ denotes the transmission power, $P_{\mathcal{L}}$ shows the distance-based path-loss, $\sigma_{\mathrm{n}}^{2}$ indicates the noise power and $\gamma[t]$ represents the normalized channel power gain of the considered unit-variance Nakagami- $m$ block fading channel with the probability density function $(\mathrm{PDF})^{1}[26]$

$$
f_{\gamma}(\gamma)=\frac{m^{m} \gamma^{m-1}}{\Gamma(m)} \mathrm{e}^{-m \gamma}
$$

where $\Gamma(z)=\int_{0}^{\infty} w^{z-1} \mathrm{e}^{-w} d w$ is the Gamma function [27]. To be specific, the Nakagami- $m$ fading distribution is parameterized by the fading parameter $m$ [1]. For $m=1$, the distribution matches Rayleigh fading, whereas, for $m=\frac{(K+1)^{2}}{(2 K+1)}$, the distribution is approximately Rician fading with parameter $K$ [1]. The case of $m \rightarrow \infty$ describes the Additive White Gaussian Noise (AWGN) channels [1].

\section{B. Problem Formulation}

Since EE and SE have to be simultaneously optimized over a feasible set determined by constraint functions [28], how to balance them falls into the scope of an MOP. To get rid of the different measurements and orders of magnitude of $\mathrm{EE}$ and SE, we normalize them with two normalization values, $\Psi_{\mathrm{EE}}$ and $\Psi_{\mathrm{SE}}$, respectively. The normalized MOP is, hence, formulated as:

$$
\begin{aligned}
Q 1: & \max \frac{\mathrm{EE}}{\Psi_{\mathrm{EE}}} \quad \text { and } \quad \max \frac{\mathrm{SE}}{\Psi_{\mathrm{SE}}} \\
& \text { subject to: } \quad \bar{P}_{\mathrm{t}} \leq P_{\max },
\end{aligned}
$$

where $\bar{P}_{\mathrm{t}}=\mathbb{E}\left[P_{\mathrm{t}}[t]\right]$ indicates the expectation of the transmission power and $P_{\max }$ denotes the average input power limit. $\Psi_{\mathrm{EE}}$ and $\Psi_{\mathrm{SE}}$ are assumed to be the EE and SE values achieved at the same normalization factor, defined by $P_{\text {norm }}$. In more details, $\Psi_{\mathrm{EE}}=\left.\mathrm{EE}\right|_{\overline{P_{\mathrm{t}}}=P_{\text {norm }}}$ and $\Psi_{\mathrm{SE}}=\left.\mathrm{SE}\right|_{\overline{P_{\mathrm{t}}}=P_{\text {norm }}}$.

Since EE is generally defined as the ratio of SE to the total power expenditure, the inverse of the two functions in problem $Q 1$ can be minimized to make $\mathrm{SE}$ as the common denominator, yielding

$$
\begin{aligned}
Q 2: & \min \frac{\Psi_{\mathrm{EE}}}{\mathrm{EE}} \quad \text { and } \quad \min \frac{\Psi_{\mathrm{SE}}}{\mathrm{SE}} \\
& \text { subject to: } \quad \bar{P}_{\mathrm{t}} \leq P_{\max } .
\end{aligned}
$$

Lemma 1: The MOP, $Q 2$, is equivalent to the MOP $Q 1$.

Proof: The proof is provided in Appendix A.

For an MOP, instead of having a single global solution, a set of points which all fit Pareto optimality is provided. To be specific, Pareto optimal sets are solutions that cannot be improved in one objective function without deteriorating the performance in at least one of the rest of objective functions. Lemma 1 implies that if a point is Pareto optimal for problem $Q 2$, it also belongs to the Pareto optimal set for problem $Q 1$, and vice-versa.

\footnotetext{
${ }^{1}$ The block index $t$ is omitted for simplicity.
} 
In order to solve the MOP Q2 and to achieve the Pareto optimal solutions, one general way is to convert the MOP into an SOP, using weighted sum method [29], [30]. As such, the optimization problem Q2 can be transformed into:

$$
\begin{aligned}
& Q 3: \quad \min w_{1} \frac{\Psi_{\mathrm{EE}}}{\mathrm{EE}}+\left(1-w_{1}\right) \frac{\Psi_{\mathrm{SE}}}{\mathrm{SE}} \\
& \text { subject to: } \bar{P}_{\mathrm{t}} \leq P_{\max },
\end{aligned}
$$

where $w_{1} \in[0,1]$ is the importance weight. Specifically, $w_{1}$ and $1-w_{1}$ represent the relative importance of the two objective functions, $\mathrm{EE}$ and $\mathrm{SE}$, respectively. When $w_{1}=0$, the tradeoff problem reduces to an SE-maximization problem, while when $w_{1}=1$, the MOP is simplified into an EEmaximization problem. In other words, the importance of EE gradually grows as $w_{1}$ increases from 0 to 1 .

In order to guarantee the Pareto optimal solutions for problem $Q 2$, we demonstrate the following theorem which describes the relationship between the weighted sum optimal point and Pareto optimal solutions of the MOP $Q 2$.

Theorem 1: The unique optimal solution $\hat{P}$ of the weighted optimization problem, $\min \sum_{i=1}^{q} w_{i} f_{i}(P), P \in\left[0, P_{\max }\right]$, for a given $\mathbf{w}=\left\{\left[w_{i}\right]_{1 \times q} \mid w_{i} \in[0,1], \sum_{i=1}^{q} w_{i}=1\right\}$, is Pareto optimal for the MOP, $\min f_{i}(P), i=1, \ldots, q, P \in\left[0, P_{\max }\right]$.

Proof: The proof is provided in Appendix B.

Implicitly, Lemma 1 and Theorem 1 illustrate that if $\hat{P}$ is a unique optimal solution for the weighted optimization problem $Q 3$, it is Pareto optimal for the original MOP $Q 1$.

\section{LINK-LAYER EE-SE TRADEOFF}

In this section, the theory of EC and link-layer EE is introduced to incorporate the link-level delay-QoS metrics. The tradeoff performance is optimized by adaptively distributing the transmit power over time based on the channel condition and the system delay requirement. An optimal power allocation strategy for the power-unconstrained EE-EC tradeoff problem is first developed and investigated, to pave the way for powerconstrained tradeoff problem. Further, the influence of system parameters on the tradeoff performance is analyzed.

\section{A. Effective Capacity and Link-layer Energy Efficiency}

In wired networks, QoS guarantees have been extensively researched [18]. The theory of effective bandwidth was proposed to asymptotically model the stochastic behavior of a source traffic process [18]. To be specific, the effective bandwidth is defined as the minimum constant service rate required by a given arrival process for which the QoS requirement is fulfilled [18]. Inspired by these studies, the link-layer channel model can be thought of as the dual of the effective bandwidth source model. Specifically, the link-layer SE, denoted as EC, can be defined as the maximum constant arrival rate that a given service process can support in order to guarantee a certain QoS request [25].
Assuming that the Gartner-Ellis theorem [31, Pages 34-36] is satisfied, EC of an independent and identically distributed (i.i.d.) block fading channel can be expressed as [18]

$$
\mathrm{EC}=-\frac{1}{\theta T_{\mathrm{f}} B} \ln \left(\mathbb{E}\left[e^{-\theta B T_{\mathrm{f}} R[t]}\right]\right) \quad(\mathrm{b} / \mathrm{s} / \mathrm{Hz}),
$$

where the parameter $\theta(\theta>0)$ denotes the exponential decay rate of the QoS violation probability. A slower decay rate can be represented by a smaller $\theta$, which indicates that the system can tolerate a looser QoS guarantee, while a more stringent QoS requirement is expressed by a larger $\theta$.

We note that, for a dynamic queueing system with stationary ergodic arrival and service processes [32], the queue length $Q(t)$ could be non-zero. Using the large deviation theory, the queue length process $Q(t)$ converges in distribution to a steady-state queue length $Q(\infty)$ such that

$$
-\lim _{x \rightarrow \infty} \frac{\ln (\operatorname{Pr}\{Q(\infty)>x\})}{x}=\theta,
$$

where $\operatorname{Pr}\{a>b\}$ shows the probability that $a>b$ holds. This definition implies that the probability of the queue length exceeding a certain threshold $x$ decays exponentially fast as $x$ increases [33]. Taking the delay experienced by a source packet arriving at fading-block $t$, defined by $D(t)$, into consideration, the probability that the delay exceeds a maximum delay bound $D_{\max }$, can be estimated as [18]

$$
P_{\text {delay }}^{\text {out }}=\operatorname{Pr}\left\{D(t)>D_{\max }\right\} \approx \operatorname{Pr}\{Q(t)>0\} \mathrm{e}^{-\theta \mu D_{\max }},
$$

where $P_{\text {delay }}^{\text {out }}$ presents the delay-outage probability, $D_{\max }$ is in the unit of a symbol period, $\operatorname{Pr}\{Q(t)>0\}$ denotes the probability of a non-empty buffer at fading-block $t$, and can be approximated by the ratio of the constant arrival rate to the average service rate [25], [32], i.e., $\operatorname{Pr}\{Q(t)>0\} \approx \frac{\mu}{\mathbb{E}[R[t]]}$. Therefore, in order to meet a target delay-bound violation probability limit, $P_{\text {delay }}^{\text {out }}$, a source needs to limit its data rate to the maximum of $\mu$, where $\mu$ is the solution to (7).

Finally, the link-layer EE for a delay-limited system can be defined as the ratio of EC to the sum of the circuit power $P_{\mathrm{c}}$, and the average transmission power scaled by the power amplifier efficiency $\epsilon$, yielding

$$
\mathrm{EE}=\frac{\mathrm{EC}}{P_{\mathrm{c}}+\frac{1}{\epsilon} \bar{P}_{\mathrm{t}}}, \quad 0 \leq \epsilon \leq 1 .
$$

\section{B. Optimal Power Allocation}

Using (4a)-(4b) and (8), the link-layer EE-EC tradeoff problem can be expressed as

$$
\begin{aligned}
& Q 5: \min w_{1} \frac{\Psi_{\mathrm{EE}}\left(P_{\mathrm{c}}+\frac{1}{\epsilon} \bar{P}_{\mathrm{t}}\right)}{\mathrm{EC}}+\left(1-w_{1}\right) \frac{\Psi_{\mathrm{EC}}}{\mathrm{EC}} \\
& \text { subject to: } \bar{P}_{\mathrm{t}} \leq P_{\max },
\end{aligned}
$$

where $\Psi_{\mathrm{EC}}$ is the normalization value for $\mathrm{EC}$, which is defined as the EC value achieved at the normalization factor, $P_{\text {norm }}$, e.g., $\Psi_{\mathrm{EC}}=\left.\mathrm{EC}\right|_{\overline{P_{\mathrm{t}}}=P_{\text {norm }}}$. 
Replacing EC in (9a) with (1) and (5), the EE-EC tradeoff problem can be transformed into

$$
\begin{gathered}
Q 6: \min _{P_{\mathrm{r}} \geq 0} \frac{w_{1} \Psi_{\mathrm{EE}} K_{\ell}\left(P_{\mathrm{c}_{\mathrm{r}}}+\frac{1}{\epsilon} \overline{P_{\mathrm{r}}}\right)+\left(1-w_{1}\right) \Psi_{\mathrm{EC}}}{-\frac{1}{\theta T_{\mathrm{f}} B} \ln \left(\mathbb{E}_{\gamma}\left[\left(1+P_{\mathrm{r}} \gamma\right)^{-\alpha(\theta)}\right]\right)} \\
\quad \text { subject to: } \overline{P_{\mathrm{r}}} \leq \frac{P_{\max }}{K_{\ell}},
\end{gathered}
$$

where $\mathbb{E}_{\gamma}[\cdot]$ indicates the expectation over the PDF of $\gamma$. The scaled transmission power, $P_{\mathrm{r}}=\frac{P_{\mathrm{t}}}{K_{\ell}}$ is the optimization variable in (10a), which can be any nonnegative real value, i.e., $P_{\mathrm{r}} \geq 0$. In addition, since the fading coefficient is uncountable, the optimization variable, which is adapted to the fading coefficient, also forms an uncountable set. $\overline{P_{\mathrm{r}}}=\frac{\bar{P}_{\mathrm{t}}}{K_{\ell}}$ denotes the scaled average input power, $P_{\mathrm{c}_{\mathrm{r}}}=\frac{P_{\mathrm{c}}}{K_{\ell}}$ represents the circuitto-noise power ratio, $K_{\ell}=P_{\mathcal{L}} \sigma_{\mathrm{n}}^{2}$, and $\alpha(\theta)=\frac{\theta T_{\mathrm{f}} B}{\ln 2}$. After deleting the negative constant, $-\frac{1}{\theta T_{\mathrm{f}} B}$, the minimization problem (10a) reduces to a maximization problem. Then, by inverting the objective function, it can be converted back into a minimization problem, yielding ${ }^{2}$

$$
\begin{aligned}
Q 7: \min _{P_{\mathrm{r}} \geq 0} \frac{\ln \left(\mathbb{E}_{\gamma}\left[\left(1+P_{\mathrm{r}} \gamma\right)^{-\alpha(\theta)}\right]\right)}{w_{1} \Psi_{\mathrm{EE}_{\mathrm{r}}}\left(P_{\mathrm{c}_{\mathrm{r}}}+\frac{1}{\epsilon} \overline{P_{\mathrm{r}}}\right)+\left(1-w_{1}\right) \Psi_{\mathrm{EC}}} \\
\text { subject to: } \overline{P_{\mathrm{r}}} \leq \frac{P_{\max }}{K_{\ell}},
\end{aligned}
$$

where $\Psi_{\mathrm{EE}_{\mathrm{r}}}=\Psi_{\mathrm{EE}} K_{\ell}$.

1) Optimum Power Allocation With No Input Power Constraint: In this section, the unconstrained SOP is tackled to pave the way for the optimal power allocation strategy of the power-constrained SOP. Hence, we start by investigating the properties of the case with a predetermined importance weight $w_{1}$, which are summarized in the following theorem.

Theorem 2: For a predetermined importance weight, the objective function $U 7$ in the tradeoff formulation $Q 7$ has the following properties:

1) $U 7$ is continuously differentiable and strictly quasiconvex in $\overline{P_{\mathrm{r}}}$,

2) $U 7$ first decreases and then increases with $\overline{P_{\mathrm{r}}}$, which turns out to be a cup shape curve,

3)

$$
U 7^{\prime}\left\{\begin{array}{l}
>0 \text { if } U 7<\frac{\epsilon}{w_{1} \Psi_{\mathrm{EE}_{\mathrm{r}}}} f\left(\overline{P_{\mathrm{r}}}\right)^{\prime} \\
=0 \text { if } U 7=\frac{\epsilon}{w_{1} \Psi_{\mathrm{EE}_{\mathrm{r}}}} f\left(\overline{P_{\mathrm{r}}}\right)^{\prime}, \\
<0 \text { if } U 7>\frac{\epsilon}{w_{1} \Psi_{\mathrm{EE}_{\mathrm{r}}}} f\left(\overline{P_{\mathrm{r}}}\right)^{\prime}
\end{array}\right.
$$

\footnotetext{
${ }^{2}$ The objective function in problem $Q 7$ is similar to equation (4) developed in [24]. The difference is the second addend and the introduced adjustable parameters in the denominator of (11a).
}

where $f\left(\overline{P_{\mathrm{r}}}\right)=\ln \left(\mathbb{E}_{\gamma}\left[\left(1+P_{\mathrm{r}} \gamma\right)^{-\alpha(\theta)}\right]\right), \quad U 7^{\prime}=\frac{\mathrm{d} U 7}{\mathrm{~d} \overline{P_{\mathrm{r}}}}$, and $f\left(\overline{P_{\mathrm{r}}}\right)^{\prime}=\frac{\mathrm{d} f\left(\overline{P_{\mathrm{r}}}\right)}{\mathrm{d} \overline{P_{\mathrm{r}}}}$.

Proof: The proof is provided in Appendix C.

In Theorem 2, Property 1) reveals the differentiability of (11a) and guarantees the existence and uniqueness of the global minimum, for a predetermined weight value. Property 2) indicates that the global optimum is always achieved at a finite power value. From Property 2) and Property 3), we notice that when $\overline{P_{\mathrm{r}}} \rightarrow 0, U 7^{\prime}<0$, which means now $U 7>$ $\frac{\epsilon}{w_{1} \Psi_{\mathrm{EE}_{\mathrm{r}}}} f\left(\overline{P_{\mathrm{r}}}\right)^{\prime}$. With $\overline{P_{\mathrm{r}}}$ increasing, $U 7$ gradually declines until it equals to $\frac{\epsilon}{w_{1} \Psi_{\mathrm{EE}_{\mathrm{r}}}} f\left(\overline{P_{\mathrm{r}}}\right)^{\prime}$. After that point, $U 7$ starts to increase with $\overline{P_{\mathrm{r}}}$. Basically, Property 3 ) connects the sign of the first derivative with the relative size of $U 7$ and the scaled first derivative of $f\left(\overline{P_{\mathrm{r}}}\right)$.

Further, we provide Lemma 2 to solve problem $Q 7$.

Lemma 2: A ratio problem $(P): \min _{x \in S} \frac{f(x)}{g(x)}$, where $f$ is convex and $g$ is affine and positive, $f, g: S \rightarrow R, S \subseteq R^{n}$, can be transformed into a convex program

$$
\begin{aligned}
\left(P^{\prime}\right): \min _{y / \phi \in S} \phi f(y / \phi) \\
\quad \text { subject to: } \phi g(y / \phi)=1,
\end{aligned}
$$

by using the Charnes-Cooper transformation [34] $y=$ $\frac{1}{g(x)} x, \phi=\frac{1}{g(x)}$, where $\phi>0$.

Proof: The proof is provided in Appendix D.

According to Lemma 2, the minimization problem (11a)(11b) reduces to the following equivalent problem $Q 8$, by applying the Charnes-Cooper transformation and one further step of substitution ${ }^{3}$.

$$
\begin{aligned}
Q 8: \min _{P_{\mathrm{r}} \geq 0} \phi \ln ( & \left.\mathbb{E}_{\gamma}\left[\left(1+P_{\mathrm{r}} \gamma\right)^{-\alpha(\theta)}\right]\right) \\
\text { subject to: } & \phi\left(w_{1} \Psi_{\mathrm{EE}_{\mathrm{r}}}\left(P_{\mathrm{C}_{\mathrm{r}}}+\frac{1}{\epsilon} \overline{P_{\mathrm{r}}}\right)\right. \\
& \left.+\left(1-w_{1}\right) \Psi_{\mathrm{EC}}\right)=1 .
\end{aligned}
$$

We note that problem $Q 8$ is not jointly convex in $P_{\mathrm{r}}$ and $\phi$. But, by regarding $\phi$ as a parameter, problem $Q 8$ becomes a convex program in $P_{\mathrm{r}}$, since the objective function is convex [24] and the constraint is an affine function in $P_{\mathrm{r}}$. The KKT conditions are, hence, sufficient and necessary for the optimal solution. Set $\lambda \in \mathbb{R}_{+}, \mathbb{R}_{+} \equiv[0, \infty]$ as the Lagrange multiplier, the Lagrangian function can be expressed as

$$
\begin{aligned}
& \mathcal{L}\left(P_{\mathrm{r}}, \lambda\right)=\phi \ln \left(\mathbb{E}_{\gamma}\left[\left(1+P_{\mathrm{r}} \gamma\right)^{-\alpha(\theta)}\right]\right) \\
& +\lambda\left(\phi\left(w_{1} \Psi_{\mathrm{EE}_{\mathrm{r}}}\left(P_{\mathrm{C}_{\mathrm{r}}}+\frac{1}{\epsilon} \overline{P_{\mathrm{r}}}\right)+\left(1-w_{1}\right) \Psi_{\mathrm{EC}}\right)-1\right) .
\end{aligned}
$$

\footnotetext{
${ }^{3}$ The Charnes-Cooper transformation is first utilized to achieve the convex program $\left(P^{\prime}\right)$, then problem $Q 8$ is derived by substituting $x=\frac{y}{\phi}$ in problem $\left(P^{\prime}\right)$.
} 
The KKT condition $\frac{\partial \mathcal{L}\left(P_{\mathrm{r}}, \lambda\right)}{\partial P_{\mathrm{r}}}=0$ can be expanded as

$$
\begin{aligned}
& \alpha(\theta) \int_{0}^{\infty}\left(1+P_{\mathrm{r}} \gamma\right)^{-\alpha(\theta)-1} \gamma f(\gamma) \mathrm{d} \gamma \\
= & \frac{\lambda w_{1} \Psi_{\mathrm{EE}_{\mathrm{r}}}}{\epsilon} \mathbb{E}_{\gamma}\left[\left(1+P_{\mathrm{r}} \gamma\right)^{-\alpha(\theta)}\right] \int_{0}^{\infty} f(\gamma) \mathrm{d} \gamma .
\end{aligned}
$$

Finally, it can be expressed as

$\alpha(\theta) \gamma\left(1+P_{\mathrm{r}}^{*} \gamma\right)^{-\alpha(\theta)-1}=\frac{\lambda w_{1} \Psi_{\mathrm{EE}_{\mathrm{r}}}}{\epsilon} \mathbb{E}_{\gamma}\left[\left(1+P_{\mathrm{r}}^{*} \gamma\right)^{-\alpha(\theta)}\right]$,

and the optimum power distribution scheme can be found as

$$
P_{\mathrm{r}}^{*}=\left[\frac{\alpha(\theta)^{\frac{1}{1+\alpha(\theta)}}}{\left(w_{1} v\right)^{\frac{1}{1+\alpha(\theta)}} \gamma^{\frac{\alpha(\theta)}{1+\alpha(\theta)}}}-\frac{1}{\gamma}\right]^{+},
$$

where $v=\frac{\lambda \Psi_{\mathrm{EE}_{\mathrm{r}}}}{\epsilon} \mathbb{E}_{\gamma}\left[\left(1+P_{\mathrm{r}}^{*} \gamma\right)^{-\alpha(\theta)}\right]$ is referred to as the scaled-Lagrangian-multiplier and $[x]^{+}=\max \{0, x\}$.

Now the optimal value of $\phi$ can be found. Since all unknowns have been expressed as explicit functions of $v$, this reduces to finding $v^{*}$ from the following equation

$$
\begin{aligned}
& \nabla_{\phi} \mathcal{L}=\ln \left(\mathbb{E}_{\gamma}\left[\left(1+P_{\mathrm{r}}^{*} \gamma\right)^{-\alpha(\theta)}\right]\right) \\
& +\lambda\left(w_{1} \Psi_{\mathrm{EE}_{\mathrm{r}}}\left(P_{\mathrm{c}_{\mathrm{r}}}+\frac{1}{\epsilon} \overline{P_{\mathrm{r}}^{*}}\right)+\left(1-w_{1}\right) \Psi_{\mathrm{EC}}\right)=0 .
\end{aligned}
$$

By substituting the power allocation (14) into (15), the optimal value for $v$ (referred to as $v^{*}$ ) can be easily found using the following equation

$$
\begin{aligned}
& \Psi_{\mathrm{EE}_{\mathrm{r}}} \mathbb{E}_{\gamma}\left[\left(1+\left[\frac{(\gamma \alpha(\theta))^{\frac{1}{1+\alpha(\theta)}}}{\left(w_{1} v^{*}\right)^{\frac{1}{1+\alpha(\theta)}}}-1\right]^{+}\right)^{-\alpha(\theta)}\right] \\
& \times \ln \left(\mathbb{E}_{\gamma}\left[\left(1+\left[\frac{(\gamma \alpha(\theta))^{\frac{1}{1+\alpha(\theta)}}}{\left(w_{1} v^{*}\right)^{\frac{1}{1+\alpha(\theta)}}}-1\right]^{+}\right)^{-\alpha(\theta)}\right]\right)+\epsilon \nu^{*} \\
& \times\left(w_{1} \Psi_{\mathrm{EE}_{\mathrm{r}}}\left(P_{\mathrm{c}_{\mathrm{r}}}+\frac{1}{\epsilon} \mathbb{E}_{\gamma}\left[\frac{\alpha(\theta)^{\frac{1}{1+\alpha(\theta)}}}{\left(w_{1} v^{*}\right)^{\frac{1}{1+\alpha(\theta)}} \gamma^{\frac{\alpha(\theta)}{1+\alpha(\theta)}}}-\frac{1}{\gamma}\right]^{+}\right)\right. \\
& \left.+\left(1-w_{1}\right) \Psi_{\mathrm{EC}}\right)=0 .
\end{aligned}
$$

For the Nakagami- $m$ fading channel, the expectations in (16) can be calculated by (18a) and (18b), as shown at the bottom of the next page, wherein $\Gamma(a, x)=\int_{x}^{\infty} z^{a-1} \mathrm{e}^{-z} d z$ is the upper incomplete gamma function and $\mathrm{E}_{1}(x)=\int_{x}^{\infty} \frac{\mathrm{e}^{-z}}{z} d z$ indicates the exponential integral $[27]^{4}$. After replacing expectations with the closed-form expressions, the optimal value for $v$, i.e., $v^{*}$, can be solved from (16) using root-finding functions,

\footnotetext{
${ }^{4}$ It is assumed that the path of integration excludes the origin and does not cross the negative real axis [27]
}

e.g., fzero in Matlab. The optimal operating input power level $P_{\mathrm{t}}^{*}$ can then be found by inserting $v^{*}$ into 18 a, namely

$$
\overline{P_{\mathrm{t}}^{*}}=K_{\ell} \times\left.\overline{P_{\mathrm{r}}^{*}}\right|_{\nu=\nu^{*}}
$$

Since the channel is assumed to be stationary and ergodic, henceforth, its average will not be affected by the shift in the time origin. Also, the pointwise mapping between $P_{\mathrm{r}}$ and $\gamma$ is fixed for each fading realization and is determined by the power allocation policy that depends on $\overline{P_{\mathrm{r}}}$.

The above equations conclude the power-unconstrained EEEC tradeoff solution. Now we provide the following analysis to pave the way for power-constrained EE-EC tradeoff problem, that is presented in next Section. Let us assume the optimal average power $\overline{P_{\mathrm{t}}^{*}}$ which solves the power-unconstrained tradeoff problem is found. Then, the power-unconstrained EE-EC tradeoff problem simplifies into an EC-maximization problem with an input power constraint, yielding

$$
\begin{aligned}
\max _{P_{\mathrm{r}} \geq 0} & -\frac{1}{\theta T_{\mathrm{f}} B} \ln \left(\mathbb{E}_{\gamma}\left[\left(1+P_{\mathrm{r}} \gamma\right)^{-\alpha(\theta)}\right]\right) \\
\text { subject to }: & \overline{P_{\mathrm{r}}} \leq \frac{\overline{P_{\mathrm{t}}^{*}}}{K_{\ell}} .
\end{aligned}
$$

2) Optimal Power Allocation Under Average Input Power Constraint: In this section, we aim to solve the optimization problem (11a)-(11b) using the results of Subsection III-B1. After the unique optimum average power value $\overline{P_{\mathrm{t}}^{*}}$ for the power-unconstrained problem is calculated, we need to compare $\overline{P_{\mathrm{t}}^{*}}$ and the input average power limit $P_{\max }$. If $\overline{P_{\mathrm{t}}^{*}} \leq P_{\max }$, it means that now the system has enough power to support the optimal tradeoff performance in Subsection III-B1. Otherwise, $P_{\mathrm{t}}^{*} \geq P_{\max }$ means that $P_{\max }$ is too small to support the power allocation strategy (14)-(18b) and the system has to operate at $P_{\max }$ to fulfill the tradeoff requirement. Therefore, the operational input average power value becomes $\min \left(\bar{P}_{\mathrm{t}}^{*}, P_{\max }\right)$.

Hence, the power-constrained EE-EC tradeoff problem in (11a)-(11b) simplifies to an EC-maximization problem with two input power constraints, yielding

$$
\begin{aligned}
\max _{P_{\mathrm{r}} \geq 0} & -\frac{1}{\theta T_{\mathrm{f}} B} \ln \left(\mathbb{E}_{\gamma}\left[\left(1+P_{\mathrm{r}} \gamma\right)^{-\alpha(\theta)}\right]\right) \\
\text { subject to: } & \overline{P_{\mathrm{r}}} \leq \frac{\overline{P_{\mathrm{t}}^{*}}}{K_{\ell}} \\
\overline{P_{\mathrm{r}}} & \leq \frac{P_{\max }}{K_{\ell}} .
\end{aligned}
$$

The optimal power allocation to solve (11a)-(11b) is according to (14), wherein, optimal $v^{*}$ is found such that $\left.K_{\ell} \bar{P}_{\mathrm{r}}\right|_{v=v^{*}}=$ $\min \left(\overline{P_{\mathrm{t}}^{*}}, P_{\max }\right)$.

To summarize, the Pseudocode of the optimal power allocation process to solve (11a)-(11b) is illustrated in Table I.

Furthermore, the optimal power allocation strategy (14)(18b) has the following properties:

\section{Properties 1:}

1) The proposed optimal solution (14)-(18b) for every given weight value, is sufficient for the Pareto optimal set of the original EE-EC MOP $Q 1$. 
2) The proposed optimal solution (14)-(18b) includes the optimal power allocation strategy for the link-layer EEmaximization problem (when $w_{1}=1$ ) and also the one for EC-maximization problem (when $w_{1}=0$ ), as extreme cases.

3) When $\theta \rightarrow 0$, EC is equivalent to the ergodic capacity. For the weighted physical-layer EE-SE tradeoff problem, the optimum power allocation strategy is the traditional water-filling approach, with the water level to be chosen so that the maximum tradeoff performance can be achieved [24].

4) When $\theta \rightarrow \infty$, EC is equivalent to the zero-outage capacity, and the optimum power allocation strategy is to maintain a constant received SNR, at a level that maximizes the tradeoff performance [35].

In more details, we note that the unique optimal solution of $Q 8$ with a predetermined importance weight, is sufficient for the optimal solution of the weighted tradeoff problem $Q 7$ [36] [37]. Then, by applying Lemma 1, Theorem 1 and Theorem 2, one can show that the optimal power allocation strategy (14)(18b) for every determined weight value, is sufficient for the Pareto optimal set of the original EE-EC MOP $Q 1$.

Furthermore, the optimal solution (14)-(18b) is similar to the optimal power allocation strategy for the link-layer EEmaximization problem in [24], with a different value of the optimal scaled-Lagrangian-multiplier $v^{*}$. When $w_{1}=1$, we note that the proposed optimal solution (14) equals to the one developed in [24]. It means that the optimal solution in [24] is a special case of the optimal power allocation strategy for the weighted EE-EC tradeoff problem in this paper. Especially, in [24], the optimal operational average power equals to $\min \left(P_{\mathrm{EE}}^{*}, P_{\max }\right)$. For a typical EE-EC tradeoff problem, the optimal average power level will remain between $\left[P_{\mathrm{EE}}^{*}, P_{\mathrm{max}}\right]$.

When $\theta \rightarrow 0$, by following similar steps, the optimal power allocation strategy for weighted tradeoff problem can be derived as

$$
P_{\mathrm{r}}=\left(\frac{1}{\rho}-\frac{1}{\gamma}\right)^{+},
$$

which is the well-known water-filling approach and $\rho$ can be found from the KKT condition

$$
\begin{gathered}
\mathbb{E}_{\gamma}\left[\left(\ln \left(\frac{\gamma}{\rho}\right)\right)^{+}\right]-\rho\left(\left(\epsilon P_{\mathrm{c}_{\mathrm{r}}}+\mathbb{E}_{\gamma}\left[\left(\frac{1}{\rho}-\frac{1}{\gamma}\right)^{+}\right]\right)\right. \\
\left.+\frac{\epsilon\left(1-w_{1}\right) \Psi_{\mathrm{EC}}}{w_{1} \Psi_{\mathrm{EE}_{\mathrm{r}}}}\right)=0 .
\end{gathered}
$$

When $\theta \rightarrow \infty$, a system with extremely stringent delay requirement is considered, which means in this case, the effective capacity is the same as the zero-outage capacity [24].

\section{The Effects of $w_{1}, P_{\text {norm }}, P_{\mathrm{c}_{\mathrm{r}}}$ and $\epsilon$ on the EE-EC tradeoff}

From (14)-(18b), we notice that the tradeoff optimal power value can be influenced by four factors, which are the importance weight $w_{1}$, normalization factor $P_{\text {norm }}$, scaled circuit

$$
\begin{aligned}
& \overline{P_{\mathrm{r}}^{*}}
\end{aligned}
$$

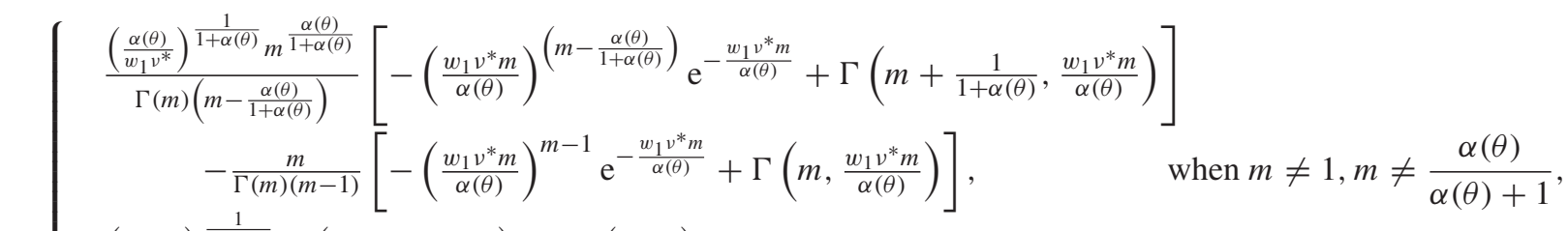

$$
\begin{aligned}
& =\left\{\left(\frac{\alpha(\theta)}{w_{1} v^{*}}\right)^{\frac{1}{1+\alpha(\theta)}} \Gamma\left(\frac{1}{1+\alpha(\theta)}, \frac{w_{1} v^{*}}{\alpha(\theta)}\right)-\mathrm{E}_{1}\left(\frac{w_{1} v^{*}}{\alpha(\theta)}\right), \quad \text { when } m=1,\right. \\
& \frac{\left(\frac{\alpha(\theta)}{w_{1} v^{*}}\right)^{\frac{1}{\alpha(\theta)+1}}\left(\frac{\alpha(\theta)}{\alpha(\theta)+1}\right)^{\frac{\alpha(\theta)}{\alpha(\theta)+1}}}{\Gamma\left(\frac{\alpha(\theta)}{\alpha(\theta)+1}\right)} \mathrm{E}_{1}\left(\frac{w_{1} v^{*}}{1+\alpha(\theta)}\right) \\
& +\frac{\alpha(\theta)}{\Gamma\left(\frac{\alpha(\theta)}{\alpha(\theta)+1}\right)}\left[-\mathrm{e}^{-\frac{w_{1} v^{*}}{\alpha(\theta)+1}}\left(\frac{w_{1} v^{*}}{\alpha(\theta)+1}\right)^{-\frac{1}{\alpha(\theta)+1}}+\Gamma\left(\frac{\alpha(\theta)}{\alpha(\theta)+1}, \frac{w_{1} v^{*}}{\alpha(\theta)+1}\right)\right], \quad \text { when } m=\frac{\alpha(\theta)}{\alpha(\theta)+1}, \\
& \mathbb{E}_{\gamma}\left[\left(1+P_{\mathrm{r}}^{*} \gamma\right)^{-\alpha(\theta)}\right] \\
& \int\left(\frac{w_{1} v^{*}}{\alpha(\theta)}\right)^{\frac{\alpha(\theta)}{1+\alpha(\theta)}} \frac{m^{\frac{\alpha(\theta)}{1+\alpha(\theta)}}}{\Gamma(m)\left(m-\frac{\alpha(\theta)}{1+\alpha(\theta)}\right)}\left[-\left(\frac{w_{1} v^{*} m}{\alpha(\theta)}\right)^{m-\frac{\alpha(\theta)}{1+\alpha(\theta)}} \mathrm{e}^{-\frac{w_{1} v^{*} m}{\alpha(\theta)}}\right. \\
& \left.+\Gamma\left(m+\frac{1}{1+\alpha(\theta)}, \frac{w_{1} v^{*} m}{\alpha(\theta)}\right)\right]+1-\frac{\Gamma\left(m, \frac{w_{1} v^{*} m}{\alpha(\theta)}\right)}{\Gamma(m)}, \quad \text { when } m \neq \frac{\alpha(\theta)}{\alpha(\theta)+1}, \\
& \frac{\left(\frac{w_{1} v^{*}}{\alpha(\theta)+1}\right)^{\frac{\alpha(\theta)}{1+\alpha(\theta)}}}{\Gamma\left(\frac{\alpha(\theta)}{\alpha(\theta)+1}\right)} \mathrm{E}_{1}\left(\frac{w_{1} v^{*}}{\alpha(\theta)+1}\right)+1-\frac{\Gamma\left(\frac{\alpha(\theta)}{\alpha(\theta)+1}, \frac{w_{1} v^{*}}{\alpha(\theta)+1}\right)}{\Gamma\left(\frac{\alpha(\theta)}{\alpha(\theta)+1}\right)}, \quad \text { when } m=\frac{\alpha(\theta)}{\alpha(\theta)+1}
\end{aligned}
$$


TABLE I

Power Allocation Algorithm

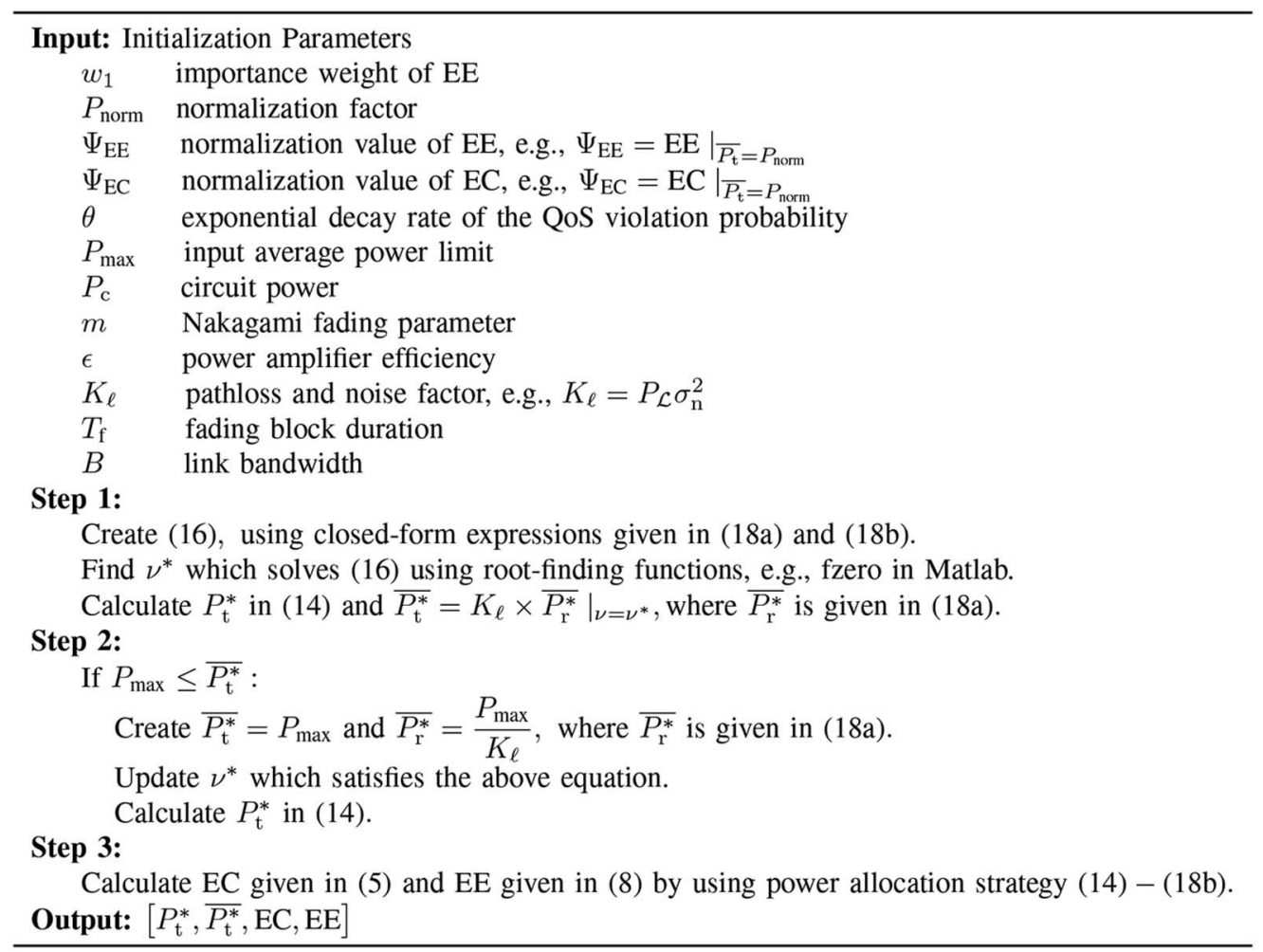

power $P_{\mathrm{c}_{\mathrm{r}}}$, and power amplifier efficiency $\epsilon$. In order to thoroughly understand the effects of these factors on the tradeoff performance, we provide the following lemmas.

Lemma 3: The average optimal power value $\overline{P_{\mathrm{t}}^{*}}$ monotonically decreases with $w_{1}$, but strictly increases with $P_{\text {norm }}$.

Proof: The proof is provided in Appendix E.

Intuitively, Lemma 3 can be clarified as follows. $\Psi_{\mathrm{EE}}$ and $\Psi_{\mathrm{EC}}$, can not only function as the normalization values, but also can be regarded as two weights. Then, the complete weights of EE and EC would be viewed as $w_{1} \Psi_{\mathrm{EE}}$ and $\left(1-w_{1}\right) \Psi_{\mathrm{EC}}$, respectively. In order to compare the relative importance of the two objective functions, we need to compare the relative weight, using

$$
\frac{\mathrm{W}_{\mathrm{EE}}}{\mathrm{W}_{\mathrm{EC}}}=\frac{w_{1} \Psi_{\mathrm{EE}}}{\left(1-w_{1}\right) \Psi_{\mathrm{EC}}}=\frac{1}{K_{\ell}\left(\frac{1}{w_{1}}-1\right)\left(P_{\mathrm{c}_{\mathrm{r}}}+\frac{1}{\epsilon} P_{\mathrm{norm}}\right)},
$$

where $\mathrm{W}_{\mathrm{EE}}=w_{1} \Psi_{\mathrm{EE}}$ and $\mathrm{W}_{\mathrm{EC}}=\left(1-w_{1}\right) \Psi_{\mathrm{EC}}$ denote the complete weights of $\mathrm{EE}$ and $\mathrm{EC}$, respectively. We notice that $\mathrm{W}_{\mathrm{EE}} / \mathrm{W}_{\mathrm{EC}}$ increases with $w_{1}$, which means that with increasing $w_{1}$, the importance of EC drops, and hence, the system prefers to sacrifice more EC to achieve better EE. Therefore, the optimum average transmit power $\overline{P_{\mathrm{t}}^{*}}$ will be shifted from $P_{\mathrm{max}}$-side to $P_{\mathrm{EE}}^{*}$-side. On the other hand, when $P_{\text {norm }}$ grows, the ratio of $\mathrm{W}_{\mathrm{EE}} / \mathrm{W}_{\mathrm{EC}}$ decreases, which means that the system prefers to improve EC, with certain deteriorations of EE. Therefore, following the same trend with $\mathrm{EC}, \overline{P_{\mathrm{t}}^{*}}$ will increase.

Lemma 3 provides a proper guideline for users to design a more flexible and favorable system, based on diverse preferences and different system requirements. For example, if the system prefers a better EC, a larger $P_{\text {norm }}$ as well as a smaller $w_{1}$ should be chosen to offer a larger optimal transmit power, and in turn, a relatively larger EC. In contrast, if a user prefers more EE, a smaller normalization factor as well as a larger $w_{1}$ will be more beneficial.

To investigate the effects of the scaled circuit power $P_{\mathrm{c}_{\mathrm{r}}}$ and the power amplifier efficiency $\epsilon$, we introduce the following lemma.

Lemma 4: The average optimal power $\overline{P_{\mathrm{t}}^{*}}$ monotonically increases with the scaled circuit power $P_{\mathrm{c}_{\mathrm{r}}}$, as well as $\epsilon$.

Proof: Following the similar proof with Lemma 3, Lemma 4 is easy to prove and it is omitted here.

\section{NumERicAl RESUlts}

In this section, we numerically investigate the impact of the normalization factor $P_{\text {norm }}$, fading severness parameter $m$, scaled circuit power $P_{\mathrm{c}_{\mathrm{r}}}$, importance weight $w_{1}$, and transmission power constraint on the link-layer EE-EC tradeoff problem for a flat block-fading channel with delay-outage probability constraints. In the following figures, we assume the fading-block duration $T_{\mathrm{f}}=2 \mathrm{~ms}$, bandwidth $B=250 \mathrm{kHz}$, input average power limit $P_{\max }=10 \mathrm{~dB}$, power amplifier efficiency $\epsilon=0.5$, fading parameter $m=1$, and the QoS exponent $\theta=10^{-2}$, unless otherwise indicated.

Fig. 2 includes the plots for EC (on the left-hand-side (LHS) y-Axis, in solid lines with markers) and EE (on the right-hand-side (RHS) y-Axis, markers only) versus importance weight $w_{1}$, for various scaled circuit power values with 


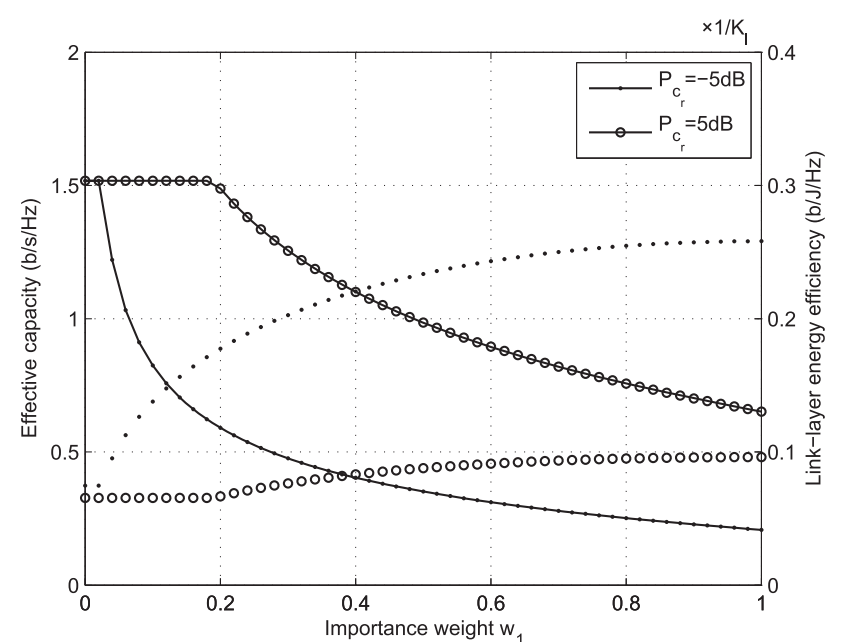

Fig. 2. EC and link-layer EE versus importance weight $w_{1}$ for various values of $P_{\mathrm{c}_{\mathrm{r}}}$ in Rayleigh fading channels.

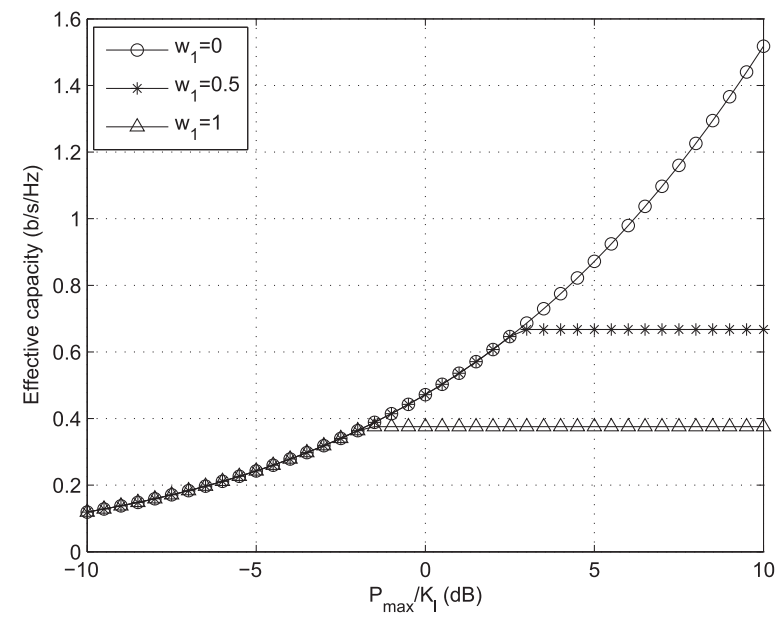

Fig. 3. EC versus scaled average input power limit $\frac{P_{\max }}{K_{\ell}}$ for various values of importance weight $w_{1}$ in Rayleigh fading channels.

normalization factor $P_{\mathrm{norm}}=0.5 P_{\mathrm{EE}}^{*}$. This figure reveals that, when $w_{1} \in[0.18,1]$, the link-layer EE increases whereas EC gradually decreases with $w_{1}$. This happens because the increase of $w_{1}$ raises the importance of $\mathrm{EE}$ and diminishes the priority of EC, which confirms our design intention. Moreover, when $w_{1} \in[0,0.18]$ and $P_{\mathrm{c}_{\mathrm{r}}}=5 \mathrm{~dB}$, there is a flat region wherein $\mathrm{EE}$ and EC remain constant. It happens because, in this region, the optimum average input power $\overline{P_{\mathrm{t}}^{*}}$ is larger than $P_{\max }$. Since the power-constrained tradeoff system performs at $\min \left(\overline{P_{\mathrm{t}}^{*}}, P_{\max }\right)$, the constant $\mathrm{EE}$ and EC versus $w_{1}$ will be observed. Furthermore, when $P_{\mathrm{c}_{\mathrm{r}}}=5 \mathrm{~dB}$, the flat region is larger than the case when $P_{\mathrm{c}_{\mathrm{r}}}=-5 \mathrm{~dB}$. In Section III-C, we proved that when $P_{\mathrm{C}_{\mathrm{r}}}$ increases, the optimum average input power $\overline{P_{\mathrm{t}}^{*}}$ will increase, which means that $\overline{P_{\mathrm{t}}^{*}}$ will remain larger than $P_{\max }$ and EC will stabilize at its maximum value for a longer period of $w_{1}$. In addition, Fig. 2 also demonstrates that, with fixed $w_{1}$, when $P_{\mathrm{C}_{\mathrm{r}}}$ rises from $-5 \mathrm{~dB}$ to $5 \mathrm{~dB}$, the value

${ }^{5}$ Here $P_{\mathrm{EE}}^{*}$ is the optimum average power level for EE-maximization problem.

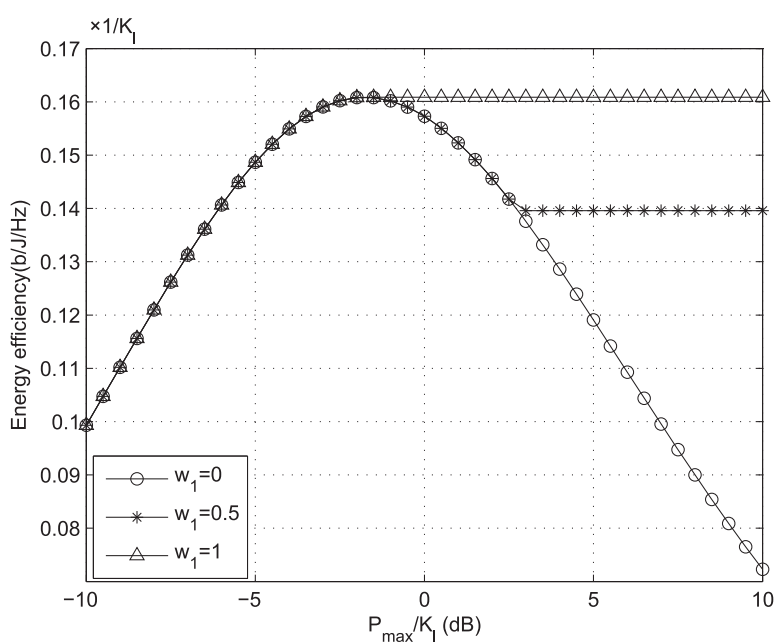

Fig. 4. Maximum achievable EE versus scaled average power limit $\frac{P_{\max }}{K_{\ell}}$ for various values of $w_{1}$ in Rayleigh fading channels.

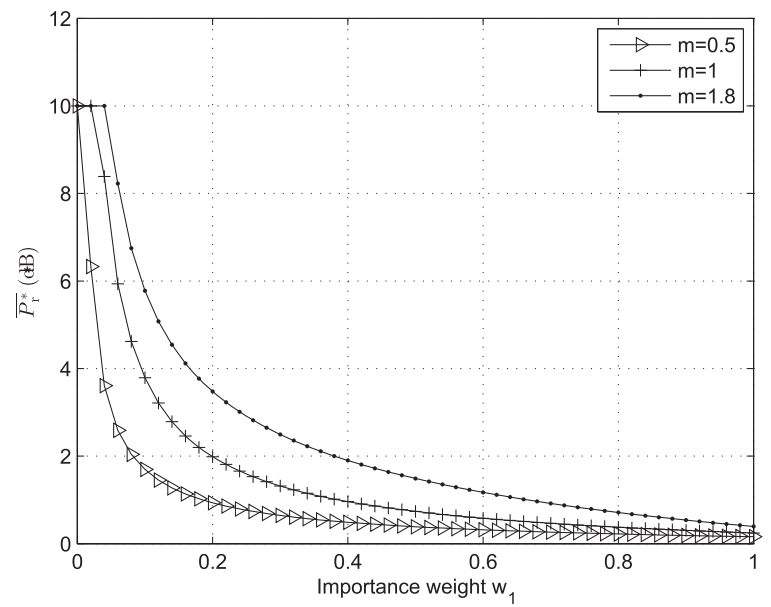

Fig. 5. Normalized optimum average power value $\overline{P_{\mathrm{r}}^{*}}$ versus importance weight $w_{1}$ for various values of fading parameter $m$.

of $\mathrm{EE}$ decreases. This is due to the fact that $\mathrm{EE}$ varies inversely with $\overline{P_{\mathrm{t}}^{*}}$, while the optimum average input power $\overline{P_{\mathrm{t}}^{*}}$ increases monotonically with the scaled circuit power $P_{\mathrm{c}_{\mathrm{r}}}$, therefore EE decreases with the circuit-to-noise power ratio $P_{\mathrm{c}_{\mathrm{r}}}$.

We plot the results of EC and EE versus $P_{\max }$, for various values of $w_{1}$ with $P_{\mathrm{C}_{\mathrm{r}}}=0 \mathrm{~dB}$ and $P_{\text {norm }}=P_{\mathrm{EE}}^{*}$ in Fig. 3 and Fig. 4, respectively. From Fig. 3, we notice that when $w_{1}=0.5$ and $w_{1}=1$, EC first continuously increases, and then it remains stable, after a break-point. This is because, for the weighted tradeoff problem with $w_{1}=0.5$ or $w_{1}=1$, the operational average power limit is settled at $\min \left(\overline{P_{\mathrm{t}}^{*}}, P_{\max }\right)$. Specifically, when $P_{\max } \leq \overline{P_{\mathrm{t}}^{*}}$, the system operates at $P_{\max }$, whereas when $P_{\max } \geq \overline{P_{\mathrm{t}}^{*}}$, the tradeoff system will not consume all the available power, but rather operates at $\overline{P_{\mathrm{t}}^{*}}$, which leads to a constant EC. These observations, however, do not apply to the case when $w_{1}=0$ which represents the ECmaximization problem. In this case, EC continuously increases with $\frac{P_{\max }}{K_{\ell}}$ while EE, shown in Fig. 4 , decreases after reaching its peak value. This is due to the fact that the allocation 


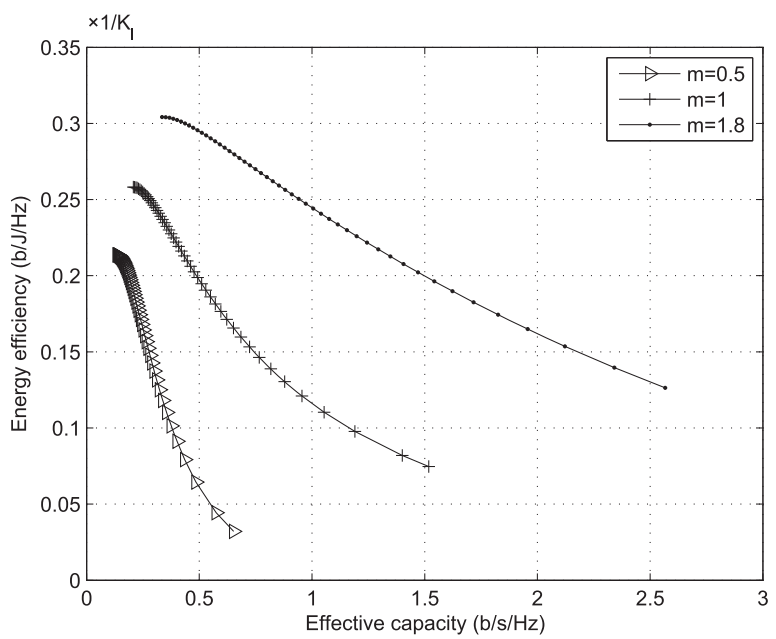

Fig. 6. Maximum achievable EE versus EC for various values of Nakagami fading parameter $m$.

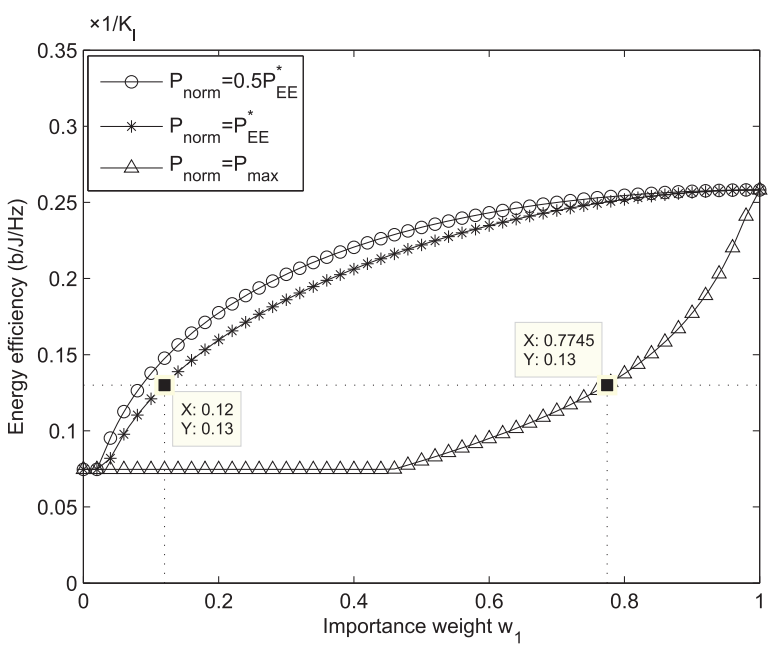

Fig. 7. Maximum achievable EE versus importance weight $w_{1}$ for various values of $P_{\text {norm }}$ in Rayleigh fading channels.

strategy for EC-maximization problem consumes the whole available input power, resulting in continuously growing EC, and simultaneously losing EE.

Similarly, from Fig. 4, we can see that when $w_{1}=0.5$, EE first increases until it reaches its peak value, at which $P_{\max }=P_{\mathrm{EE}}^{*}$, then EE gradually drops until $\overline{P_{\mathrm{t}}}=\overline{P_{\mathrm{t}}^{*}}$, after which it stabilizes. This demonstrates that the operational optimal average power, $\min \left(\overline{P_{\mathrm{t}}^{*}}, P_{\max }\right)$, is always achieved between $\left[P_{\mathrm{EE}}^{*}, P_{\max }\right]$. And, for any $P_{\max } \geq \overline{P_{\mathrm{t}}^{*}}$, the tradeoff system performs at $\overline{P_{\mathrm{t}}^{*}}$, which leads to a constant EE. In addition, for EEmaximization problem with $w_{1}=1$, Fig. 4 shows that the linklayer EE gradually increases until its peak value, achieved at $P_{\mathrm{EE}}^{*}$, after which it remains constant. This is due to the fact that the average optimal power limit for EE-maximization problem is always achieved at $\min \left(P_{\mathrm{EE}}^{*}, P_{\max }\right)$ [24], which means that when $P_{\max } \leq P_{\mathrm{EE}}^{*}$, the system operates at the most achievable power value $P_{\max }$, and then the tradeoff problem performs at the global optimal power level $P_{\mathrm{EE}}^{*}$ for any $P_{\max } \geq P_{\mathrm{EE}}^{*}$. Although Fig. 3 and Fig. 4 are plotted using link-layer capacity, the same trend can be observed in physical-layer tradeoff problem.

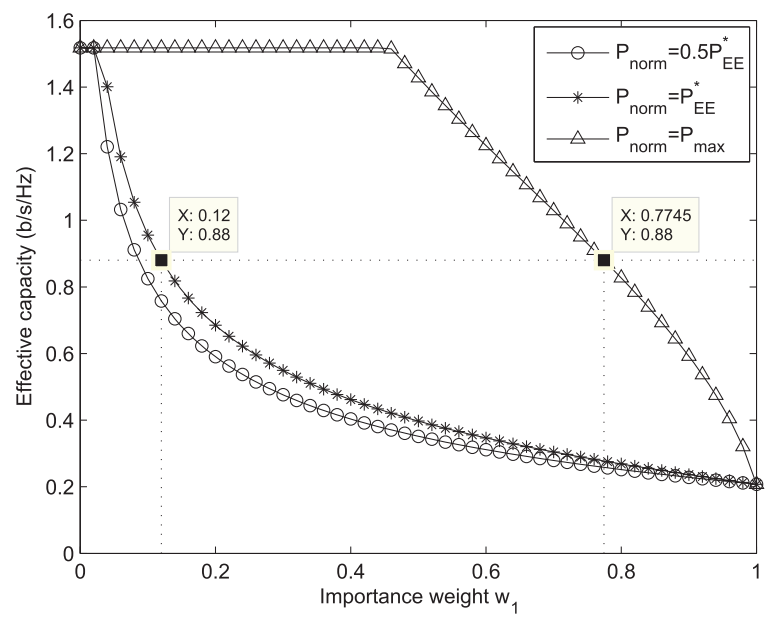

Fig. 8. EC versus importance weight $w_{1}$ for various values of normalization factor $P_{\text {norm }}$ in Rayleigh fading channels.

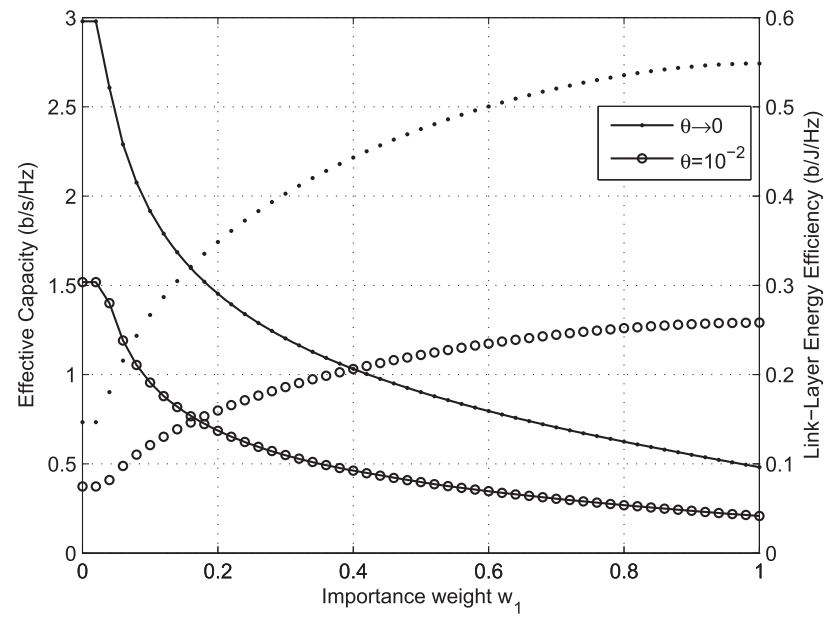

Fig. 9. EC and link-layer EE versus importance weight $w_{1}$ for various values of $\theta$ in Rayleigh fading channels.

The plot for $\overline{P_{\mathrm{r}}^{*}}$ versus $w_{1}$ for various fading parameters with $P_{\text {norm }}=0.5 P_{\mathrm{EE}}^{*}$ and $P_{\mathrm{C}_{\mathrm{r}}}=-5 \mathrm{~dB}$ is given in Fig. 5. Noting that increasing $w_{1}$ increases the importance of EE in the tradeoff problem, $\overline{P_{\mathrm{r}}^{*}}$ monotonically decreases from $P_{\max }$ to $P_{\mathrm{EE}}^{*}$. Fig. 5 further shows that, for a fixed $w_{1}$, when $m$ increases, $P_{\mathrm{r}}^{*}$ increases. This happens due to the fact that with less channel fluctuations, the probability of the received data remaining in the FIFO buffer will be dropped, and therefore, EC and $\overline{P_{\mathrm{r}}^{*}}$ will increase. We note that, when $m=1$ and $m=1.8, \overline{P_{\mathrm{r}}^{*}}$ first stabilizes at its maximum value $P_{\max }$. This is due to the fact that in this region, the optimal average power level $\overline{P_{\mathrm{t}}^{*}}$ is larger than $P_{\max }$, and therefore, the tradeoff system has to operate at $P_{\max }$.

The plots for EE versus EC, for various values of $m$ with $P_{\text {norm }}=0.5 P_{\mathrm{EE}}^{*}$ and $P_{\mathrm{c}_{\mathrm{r}}}=-5 \mathrm{~dB}$ is plotted in Fig. 6 which shows that when $m=1.8$, the MOP achieves the largest EE and EC, while the curve with the smallest $m$ provides the least values of $\mathrm{EE}$ and $\mathrm{EC}$.

Fig. 7 includes the plots for EE versus importance weight $w_{1}$, for various values of $P_{\text {norm }}$ with $P_{\mathrm{c}_{\mathrm{r}}}=-5 \mathrm{~dB}$. When $w_{1}$ is relatively large, e.g., $w_{1} \in[0.46,1]$, EE shows a consistently upward trend with the increase of $w_{1}$ for all considered values 


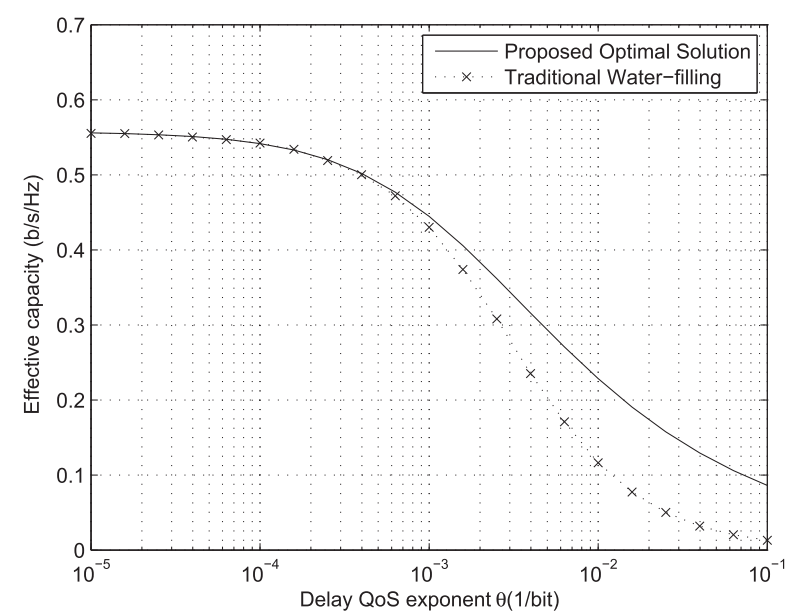

Fig. 10. EC versus delay QoS exponent $\theta$ under different power allocation policies in Rayleigh fading channels.

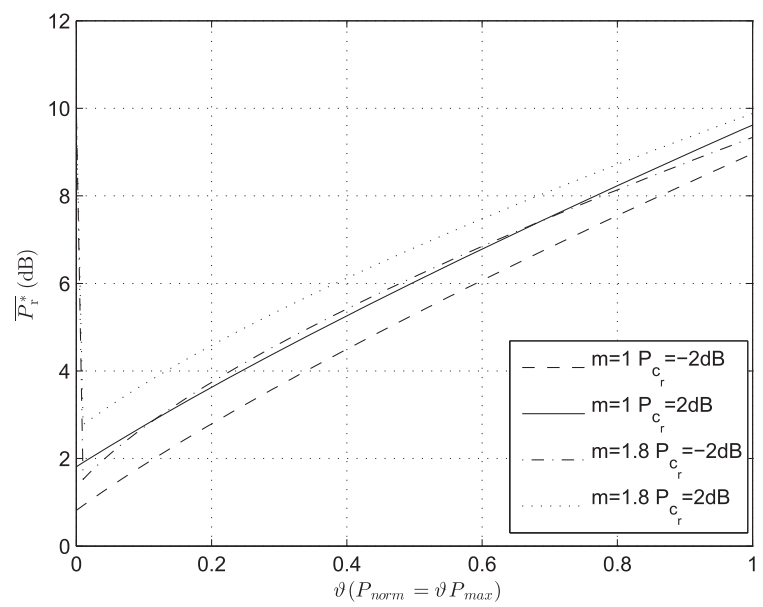

Fig. 11. Normalized optimum average power value $\overline{P_{\mathrm{r}}^{*}}$ versus $\vartheta$ for various values of fading parameter $m$ and scaled circuit power $P_{\mathrm{c}_{\mathrm{r}}}$.

of $P_{\text {norm. }}$. When $w_{1}$ is small, e.g., $w_{1} \in[0,0.46]$ and $P_{\text {norm }}=$ $P_{\max }$, EE initially remains constant until reaching a break-point, then gradually increases toward its maximum value. On the other hand, Fig. 8 shows that when $P_{\text {norm }}=P_{\max }$, EC levels off at its maximum value for a longer period of $w_{1}$, in comparison with the other EC curves with $P_{\text {norm }}=0.5 P_{\mathrm{EE}}^{*}$ and $P_{\text {norm }}=P_{\mathrm{EE}}^{*}$. This provides a guideline for an EC-desired system and indicates that with a larger normalization factor $P_{\text {norm }}$, there is a better chance to make EC remain around its maximum value for a longer scope of varying $w_{1}$. Moreover, Fig. 7 and Fig. 8 demonstrate that the ranges of EC and EE, covered by $w_{1} \in[0,1]$, are always fixed, regardless of the different definitions of $P_{\text {norm. }}$. For example, from Fig. 7, the EE curve with $P_{\mathrm{norm}}=P_{\mathrm{EE}}^{*}$, and the one with $P_{\mathrm{norm}}=P_{\max }$, achieve the same value of the scaled $\mathrm{EE}, 0.13 \mathrm{~b} / \mathrm{J} / \mathrm{Hz}$, at $w_{1}=0.12$ and $w_{1}=0.7745$, respectively. Meanwhile, in Fig. 8, EC obtained at $w_{1}=0.12, P_{\text {norm }}=P_{\mathrm{EE}}^{*}$, equals to the EC value achieved at $w_{1}=0.7745, P_{\text {norm }}=P_{\max }$.

We plot the results of EC (on the left-hand-side (LHS) yAxis, in solid lines with markers) and EE (on the right-handside (RHS) y-Axis, markers only) versus $w_{1}$, for various values

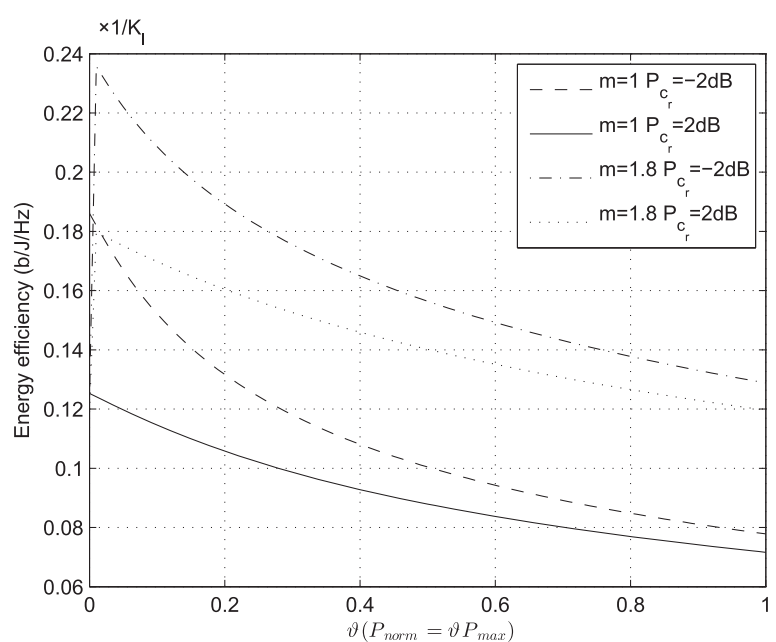

Fig. 12. Maximum achievable EE versus $\vartheta$ for various values of Nakagami fading parameter $m$ and scaled circuit power $P_{\mathrm{c}_{\mathrm{r}}}$.

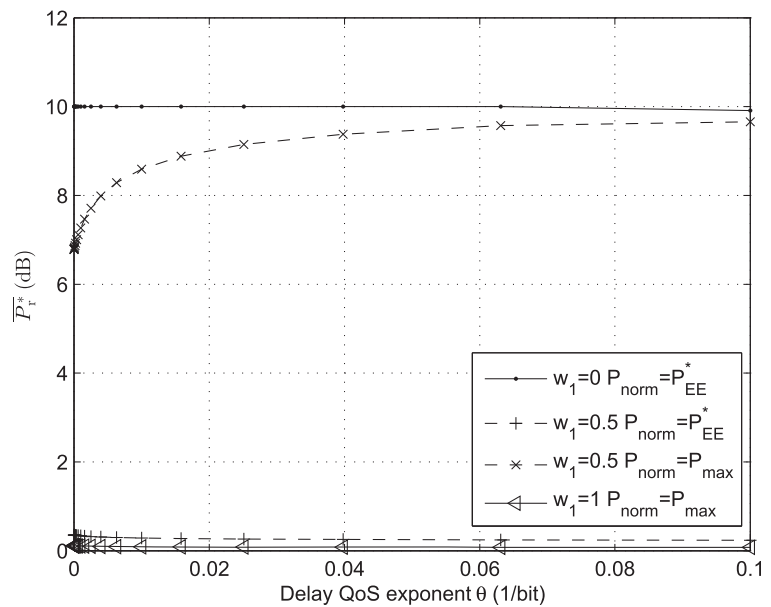

Fig. 13. Normalized optimum average power value $\overline{P_{\mathrm{r}}^{*}}$ versus $\theta$ for various values of $w_{1}$ and $P_{\text {norm }}$ in Rayleigh fading channels.

of $\theta$ with $P_{\mathrm{c}_{\mathrm{r}}}=-5 \mathrm{~dB}, P_{\text {norm }}=P_{\mathrm{EE}}^{*}$ in Fig. 9. As we discussed in Section III-B2, $\theta \rightarrow 0$ refers to a system with no delay requirement, and hence $\mathrm{EC}$ is equivalent to the ergodic capacity. For the physical-layer EE-SE tradeoff problem, no delay requirement means that the delay-outage probability can be very high. Although, from Fig. 9, EC and $\mathrm{EE}$ when $\theta \rightarrow 0$ are larger than those when $\theta=10^{-2}$, we can also notice that when $\theta$ is very small, e.g., $\theta<10^{-5}$, the delay-outage probability equals to 1 , in Fig. 14. Further, we note that the physicallayer $\mathrm{EC}$ and $\mathrm{EE}$, when $\theta \rightarrow 0$, follow the same trend with the link-layer $\mathrm{EC}$ and $\mathrm{EE}$, when $\theta=10^{-2}$.

The plot for EC versus delay QoS exponent $\theta$, under different power allocation policies, with $w_{1}=0.5, P_{\mathrm{norm}}=P_{\mathrm{EE}}^{*}$ and $P_{\mathrm{c}_{\mathrm{r}}}=-5 \mathrm{~dB}$ is included in Fig. 10. Specifically, this figure compares the EC values under the optimal link-layer power allocation solution, which is derived in this paper, and the traditional physical-layer water-filling approach. From Section III-B2, we note that when $\theta$ becomes very small, e.g., $\theta<10^{-4}$, EC approaches to ergodic capacity. In this case, the proposed optimal power allocation strategy (14)-(18b) converges to the traditional water-filling strategy. Therefore, in 


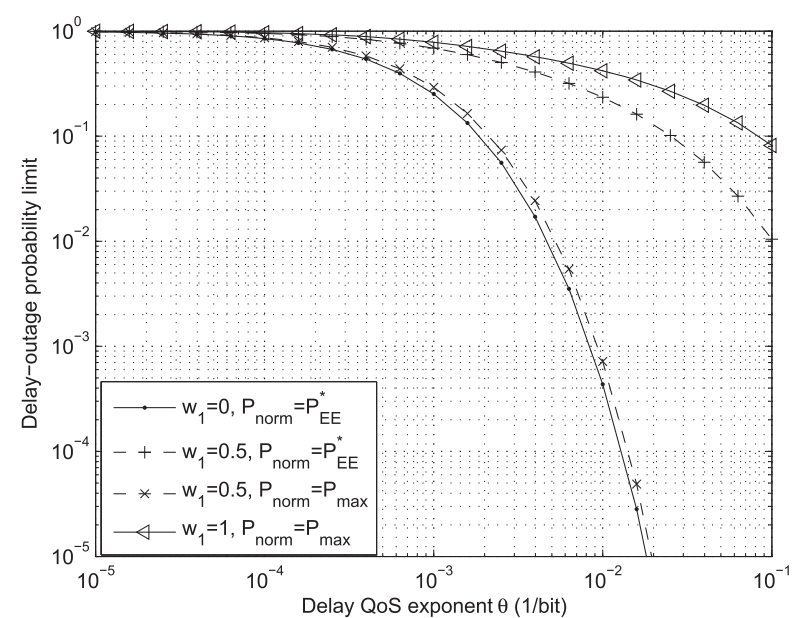

Fig. 14. Delay-outage probability versus $\theta$ for various values of $w_{1}$ and normalization factor $P_{\text {norm }}$ in Rayleigh fading channels.

Fig. 10, when $\theta<10^{-4}$, the values of EC for the two different power policies are equal. When $\theta$ becomes larger, e.g., $\theta \geq$ $10^{-3}$, which refers to a system with a stringent delay requirement, Fig. 10 indicates that the proposed link-layer optimal power allocation strategy guarantees a better performance than the traditional water-filling approach, with the water-filling performance approaching to zero when $\theta \simeq 0.1$.

Fig. 11 and Fig. 12 illustrate the optimal average power value and $\mathrm{EE}$ versus $\vartheta$, where $\vartheta$ describes the ratio of $P_{\text {norm }}$ to $P_{\max }$, i.e., $P_{\text {norm }}=\vartheta P_{\max }$, for various values of fading parameter $m$ and scaled circuit power $P_{\mathrm{c}_{\mathrm{r}}}$. Especially, a typical tradeoff system is considered and $w_{1}=0.5$. When $m=1, \overline{P_{\mathrm{r}}^{*}}$ increases, while EE decreases with $\vartheta$. This happens because when $\vartheta$ increases, $P_{\text {norm }}$ becomes larger, which indicates that the priority of EE will be decreased and the importance of EC will increase. When $m=1.8, \overline{P_{\mathrm{r}}^{*}}$ firstly plunges to its lowest value and then gradually increases with $\vartheta$, while EE first increases to its maximum value and then gradually drops with $\vartheta$. Furthermore, Fig. 11 shows that when $m$ is fixed, a system with bigger $P_{\mathrm{c}_{\mathrm{r}}}$ always has a larger $\overline{P_{\mathrm{r}}^{*}}$, which confirms our conclusion in Lemma 2.

Fig. 13 includes the plots for $\overline{P_{\mathrm{r}}^{*}}$ versus delay QoS exponent $\theta$ for various values of $w_{1}$ and $P_{\text {norm }}$, with $P_{\mathrm{C}_{\mathrm{r}}}=-10 \mathrm{~dB}$. When $w_{1}=0, \overline{P_{\mathrm{r}}^{*}}$ levels out at the maximum transmit power limit $P_{\max }$, which confirms that the EC-maximization system always consumes all the available power [25]. When $w_{1}=0.5$ and $P_{\text {norm }}=P_{\max }, \overline{P_{\mathrm{r}}^{*}}$ increases with $\theta$, until it remains stable at a certain value, which is just under $P_{\max }$. In contrast, For cases of $w_{1}=0.5$ and $P_{\text {norm }}=P_{\mathrm{EE}}^{*}$, and $w_{1}=1$ and $P_{\text {norm }}=$ $P_{\max }$, the optimum average power levels are achieved at minimal values. Furthermore, with $w_{1}=0.5, \overline{P_{\mathrm{r}}^{*}}$ is higher when $P_{\text {norm }}=P_{\max }$, comparing to a case with $P_{\text {norm }}=P_{\mathrm{EE}}^{*}$. This is due to the fact that, a larger $P_{\text {norm }}$, e.g., $P_{\text {norm }}=P_{\max }$, reduces the priority of $\mathrm{EE}$ and raises the importance of EC, which results in a larger $\overline{P_{\mathrm{r}}^{*}}$ and a smaller EE.

The delay-outage probability limit $P_{\text {delay }}^{\text {out }}$ versus delay QoS exponent for various values of $w_{1}$ and $P_{\text {norm }}$ with a maximum tolerable delay threshold $D_{\max }=500$, circuit-to-noise power ratio $P_{\mathrm{c}_{\mathrm{r}}}=-10 \mathrm{~dB}$ is illustrated in Fig. 14. This figure indicates that for loose delay-constrained systems, e.g., $\theta=$ $10^{-5}$, different values of $w_{1}$ will not affect the achievable $P_{\text {delay }}^{\text {out }}$ significantly. Also, in this case, the delay-outage probability becomes 1 , which means that the probability of the delay exceeding the maximum delay bound $D_{\max }$ is equivalent to 1 . For larger $\theta$, e.g., $\theta \geq 10^{-3}$, delay-outage probability increases with $w_{1}$. This happens because smaller $w_{1}$ represents a system which prefers EC-maximization approach. Hence, a higher EC will be achieved and the probability that the symbol delay exceeds a maximum delay-bound $D_{\max }$ will decline. Furthermore, for a fixed $\theta$, when $w_{1}=0.5$ and $P_{\text {norm }}=P_{\mathrm{EE}}^{*}$, the delay-outage probability limit is larger than that with the same $w_{1}$ and $P_{\text {norm }}=P_{\text {max }}$. This is due to the fact that a system with larger $P_{\text {norm }}$ offers a larger EC, which means that the probability of data remaining in the FIFO buffer will be dropped, and therefore, $P_{\text {delay }}^{\text {out }}$ will be smaller.

\section{CONCLUSIONS}

The optimal power distribution scheme of the link-layer EE-EC tradeoff problem for a Nakagami- $m$ fading channel with a delay violation probability constraint and an average input power limit was developed and analyzed. We proved that the proposed tradeoff formulation is continuously differentiable and strictly quasiconvex in the optimum average power level. After obtaining the optimal power allocation scheme, we proved that the proposed scheme is also sufficient for the Pareto optimal set of the original EE-EC MOP. In order to thoroughly analyze the tradeoff performance, the effects of the normalization factor, importance weight, circuit power and power amplifier efficiency were analyzed and investigated. We also provided a proper guideline on how to choose the normalization factor and importance weight to build a more favorable system toward either EE or EC.

\section{APPENDIX A \\ PROOF OF LEMMA 1}

Suppose the point $\overline{P_{\mathrm{t}}^{*}} \in\left[0, P_{\max }\right]$, is a Pareto optimal solution for problem $Q 2$ and it is not a Pareto optimal solution for problem $Q 1$. Hence, there must exist $\overline{P_{\mathrm{t}}^{\prime}}$ with $\frac{\mathrm{SE}\left(\overline{P_{\mathrm{t}}^{\prime}}\right)}{\Psi_{\mathrm{SE}}} \geq \frac{\mathrm{SE}\left(\overline{P_{\mathrm{t}}^{*}}\right)}{\Psi_{\mathrm{SE}}}, \frac{\mathrm{EE}\left(\overline{P_{\mathrm{t}}^{\prime}}\right)}{\Psi_{\mathrm{EE}}} \geq \frac{\mathrm{EE}\left(\overline{P_{\mathrm{t}}^{*}}\right)}{\Psi_{\mathrm{EE}}}$, and also at least one of the two following conditions happens: 1) $\frac{\operatorname{SE}\left(\overline{P_{\mathrm{t}}^{\prime}}\right)}{\Psi_{\mathrm{SE}}}>$ $\frac{\mathrm{SE}\left(\overline{P_{\mathrm{t}}^{*}}\right)}{\Psi_{\mathrm{SE}}}$, 2) $\frac{\mathrm{EE}\left(\overline{P_{\mathrm{t}}^{\prime}}\right)}{\Psi_{\mathrm{EE}}}>\frac{\mathrm{EE}\left(\overline{P_{\mathrm{t}}^{*}}\right)}{\Psi_{\mathrm{EE}}}$. Note that $\operatorname{SE}\left(\overline{P_{\mathrm{t}}}\right), \operatorname{EE}\left(\overline{P_{\mathrm{t}}}\right)$, for $\frac{P_{\mathrm{t}}}{P} \in\left[0, P_{\max }\right]$, are always positive, therefore, there exists $\overline{P_{\mathrm{t}}^{\prime}}$ which guarantees that $\frac{\Psi_{\mathrm{SE}}}{\mathrm{SE}\left(\overline{P_{\mathrm{t}}^{\prime}}\right)} \leq \frac{\Psi_{\mathrm{SE}}}{\operatorname{SE}\left(\overline{P_{\mathrm{t}}^{*}}\right)}, \frac{\Psi_{\mathrm{EE}}}{\operatorname{EE}\left(\overline{P_{\mathrm{t}}^{\prime}}\right)} \leq$ $\frac{\Psi_{\mathrm{EE}}}{\mathrm{EE}\left(\overline{P_{\mathrm{t}}^{*}}\right)}$, and at least one of the two following conditions happens: 1) $\frac{\Psi_{\mathrm{SE}}}{\operatorname{SE}\left(\overline{P_{\mathrm{t}}^{\prime}}\right)}<\frac{\Psi_{\mathrm{SE}}}{\operatorname{SE}\left(\overline{P_{\mathrm{t}}^{*}}\right)}$, 2) $\frac{\Psi_{\mathrm{EE}}}{\operatorname{EE}\left(\overline{P_{\mathrm{t}}^{\prime}}\right)}<\frac{\Psi_{\mathrm{EE}}}{\operatorname{EE}\left(\overline{P_{\mathrm{t}}^{*}}\right)}$. This contradicts the assumption that $\overline{P_{\mathrm{t}}^{*}}$ is a Pareto optimal solution for problem $Q 2$. This concludes the proof for Lemma 1.

${ }^{6}$ Here, $\mathrm{SE}(\bar{P})$ and $\mathrm{EE}(\bar{P})$ are defined as the $\mathrm{SE}$ and EE values achieved at certain average power $\bar{P}$. 


\section{APPENDIX B}

\section{ProOF OF THEOREM $1^{7}$}

Since $\hat{P}$ is unique optimal solution for the weighted SOP, then $\sum_{i=1}^{q} w_{i} f_{i}(\hat{P})<\sum_{i=1}^{q} w_{i} f_{i}(P), w_{i} \in[0,1], \sum_{i=1}^{q} w_{i}=1$, for all $P \in\left[0, P_{\max }\right]$. Suppose $\hat{P}$ is not a Pareto optimal solution for the MOP. Hence, there must exist $P^{\prime} \in\left[0, P_{\max }\right]$ with $f_{i}\left(P^{\prime}\right) \leq f_{i}(\hat{P})$ for all $i=1, \ldots, q$, and there is at least one $j$, such that $f_{j}\left(P^{\prime}\right)<f_{j}(\hat{P}), j=1, \ldots, q$. Multiplying by the weights, we have $w_{i} f_{i}\left(P^{\prime}\right) \leq w_{i} f_{i}(\hat{P})$ for all $i=1, \ldots, q$, and $\sum_{i=1}^{q} w_{i} f\left(P^{\prime}\right) \leq \sum_{i=1}^{q} w_{i} f(\hat{P})$. This contradicts the uniqueness assumption. Therefore, the theorem is proved.

\section{APPENDIX C}

\section{PROOF OF THEOREM 2}

Denote the sublevel set of U7 by $\mathcal{S}_{\beta}=$ $\left\{\overline{P_{\mathrm{r}}} \in\left[0, \frac{P_{\max }}{K_{\ell}}\right] \mid U 7 \leq \beta\right\}$. According to [39], $U 7$ is strictly quasiconvex in $\overline{P_{\mathrm{r}}}$ if $\mathcal{S}_{\beta}$ is strictly convex for any real number $\beta$. In more details, a set is strictly convex if any line (without the endpoints) connecting two points in the set is inside the interior of the set. In other words, the set $C$ is strictly convex if every point $c=\lambda a+(1-\lambda) b, \lambda \in(0,1), \lambda \in \mathbb{R}$, for any two points $a, b \in C, a \neq b$, is inside the interior of $C$.

Firstly, when $\beta<0$, no points exist for $U 7=\beta$. When $\beta \geq$ $0, \mathcal{S}_{\beta}$ is equivalent to

$$
\begin{aligned}
& \mathcal{S}_{\beta}=\left\{P_{\mathrm{r}} \in\left[0, \frac{P_{\mathrm{max}}}{K_{\ell}}\right] \mid \ln \left(\mathbb{E}_{\gamma}\left[\left(1+P_{\mathrm{r}} \gamma\right)^{-\alpha(\theta)}\right]\right)\right. \\
& \left.-\beta\left(w_{1} \Psi_{\mathrm{EE}_{\mathrm{r}}}\left(P_{\mathrm{C}_{\mathrm{r}}}+\frac{1}{\epsilon} \overline{P_{\mathrm{r}}}\right)+\left(1-w_{1}\right) \Psi_{\mathrm{EC}}\right) \leq 0\right\} .
\end{aligned}
$$

Since $\ln \left(\mathbb{E}_{\gamma}\left[\left(1+P_{\mathrm{r}} \gamma\right)^{-\alpha(\theta)}\right]\right)$ is strictly convex [24], and $\beta\left(w_{1} \Psi_{\mathrm{EE}_{\mathrm{r}}}\left(P_{\mathrm{c}_{\mathrm{r}}}+\frac{1}{\epsilon} \overline{P_{\mathrm{r}}}\right)+\left(1-w_{1}\right) \Psi_{\mathrm{EC}}\right)$ is affine in $\overline{P_{\mathrm{r}}}$, therefore, $\mathcal{S}_{\beta}$ is strictly convex for any real number $\beta$ and $U 7$ is strictly quasiconvex in $\overline{P_{\mathrm{r}}}$. This proves Property 1 ).

We now take the first derivative of (9a) with respect to $\overline{P_{\mathrm{r}}}$, yielding

$$
U 5^{\prime}=\frac{\frac{w_{1} \Psi_{\mathrm{EE}_{\mathrm{r}}}}{\epsilon} \mathrm{EC}-J\left(\overline{P_{\mathrm{r}}}\right) \mathrm{EC}^{\prime}}{\mathrm{EC}^{2}},
$$

where $\quad J\left(\overline{P_{\mathrm{r}}}\right)=w_{1} \Psi_{\mathrm{EE}_{\mathrm{r}}}\left(P_{\mathrm{c}_{\mathrm{r}}}+\frac{1}{\epsilon} \overline{P_{\mathrm{r}}}\right)+\left(1-w_{1}\right) \Psi_{\mathrm{EC}} \quad$ and $\mathrm{EC}^{\prime}=\frac{\mathrm{dEC}}{\mathrm{d} \overline{P_{\mathrm{r}}}}$. When $\overline{P_{\mathrm{r}}} \rightarrow 0, \mathrm{EC} \rightarrow 0, J\left(\overline{P_{\mathrm{r}}}\right)>0$ and $\mathrm{EC}^{\prime}>0$, therefore, $\left.U 5^{\prime}\right|_{\overline{P_{\mathrm{r}}} \rightarrow 0}<0$. On the other hand, when $\overline{P_{\mathrm{r}}} \rightarrow \infty$, we have

$$
\lim _{P_{\mathrm{r}} \rightarrow \infty} \frac{\frac{w_{1} \Psi_{\mathrm{EE}_{\mathrm{r}}}}{\epsilon} \mathrm{EC}}{J\left(\overline{P_{\mathrm{r}}}\right) \mathrm{EC}^{\prime}}=\lim _{\overline{P_{\mathrm{r}}} \rightarrow \infty} \frac{\frac{w_{1} \Psi_{\mathrm{EE}_{\mathrm{r}}}}{\epsilon} \mathrm{EC}^{\prime}}{\frac{w_{1} \Psi_{\mathrm{EE}_{\mathrm{r}}}}{\epsilon} \mathrm{EC}^{\prime}+J\left(\overline{P_{\mathrm{r}}}\right) \mathrm{EC}^{\prime \prime}},
$$

\footnotetext{
${ }^{7} \mathrm{~A}$ similar theorem was mentioned in [38], but the proof was not provided..
}

where $\mathrm{EC}^{\prime \prime}=\frac{\mathrm{d}^{2} \mathrm{EC}}{\mathrm{d}{\overline{P_{\mathrm{r}}}}^{2}}$. We note that $\mathrm{EC}^{\prime \prime}<0$, due to the fact that EC is strictly concave in $\overline{P_{\mathrm{r}}}$ [24]. Now, by using the fact that $J\left(\overline{P_{\mathrm{r}}}\right)>0$, one can show that the RHS of (24) is bigger than 1 , which means that $\left.U 5^{\prime}\right|_{P_{\mathrm{r}} \rightarrow \infty}>0$. Hence, when $\overline{P_{\mathrm{r}}} \rightarrow \infty$, $U 5$ is an increasing function in $\overline{P_{\mathrm{r}}}$. We note that $U 7$ is derived by canceling the negative multiplied constant in $U 5$ and then inverting the objective function. Therefore, when $\overline{P_{\mathrm{r}}} \rightarrow 0, U 7$ monotonically decreases and when $\overline{P_{\mathrm{r}}} \rightarrow \infty, U 7$ monotonically increases. This proves that $U 7$ has a cup shape curve in $\overline{P_{\mathrm{r}}}$, which completes the proof for Property 2$)$.

Now, we set $f\left(\overline{P_{\mathrm{r}}}\right)=\ln \left(\mathbb{E}_{\gamma}\left[\left(1+P_{\mathrm{r}} \gamma\right)^{-\alpha(\theta)}\right]\right)$ and take the first derivative of $U 7$ with respect to $\overline{P_{\mathrm{r}}}$ to get

$$
\begin{aligned}
U 7^{\prime} & =\lim _{\Delta \overline{P_{\mathrm{r}}} \rightarrow 0} \frac{\frac{f\left(\overline{P_{\mathrm{r}}}+\Delta \overline{P_{\mathrm{r}}}\right)}{J\left(\overline{P_{\mathrm{r}}}+\Delta \overline{P_{\mathrm{r}}}\right)}-\frac{f\left(\overline{P_{\mathrm{r}}}\right)}{J\left(\overline{P_{\mathrm{r}}}\right)}}{\Delta \overline{P_{\mathrm{r}}}} \\
& =\lim _{\Delta \overline{P_{\mathrm{r}}} \rightarrow 0} \frac{\frac{f\left(\overline{P_{\mathrm{r}}}+\Delta \overline{P_{\mathrm{r}}}\right)-f\left(\overline{P_{\mathrm{r}}}\right)}{\Delta \overline{P_{\mathrm{r}}}}-\frac{w_{1} \Psi_{\mathrm{EE}_{\mathrm{r}}}}{\epsilon} U 7}{J\left(\overline{P_{\mathrm{r}}}+\Delta \overline{P_{\mathrm{r}}}\right)} \\
& =\lim _{\Delta \overline{P_{\mathrm{r}}} \rightarrow 0} \frac{f\left(\overline{P_{\mathrm{r}}}\right)^{\prime}-\frac{w_{1} \Psi_{\mathrm{EE}_{\mathrm{r}}}}{\epsilon}}{J\left(\overline{P_{\mathrm{r}}}+\Delta \overline{P_{\mathrm{r}}}\right)} .
\end{aligned}
$$

Therefore, $\operatorname{sgn}\left(U 7^{\prime}\right)=\operatorname{sgn}\left(f\left(\overline{P_{\mathrm{r}}}\right)^{\prime}-\frac{w_{1} \Psi_{\mathrm{EE}_{\mathrm{r}}}}{\epsilon} U 7\right)$. This completes the proof of Property 3 ).

\section{APPENDIX D}

\section{Proof OF LEMMA 2}

Here, we briefly prove that problem $\left(P^{\prime}\right)$ is a convex program in $(y, \phi)$, and if $\left(y^{*}, \phi^{*}\right)$ is an optimal solution of $\left(P^{\prime}\right)$, then $x^{*}=y^{*} / \phi^{*}$ is an optimal solution of $(P)$.

Since $f$ is a convex function, therefore, for the objective function of problem $\left(P^{\prime}\right)$, we have

$$
\begin{aligned}
& \left(\lambda \phi_{1}+(1-\lambda) \phi_{2}\right) f\left(\frac{\lambda y_{1}+(1-\lambda) y_{2}}{\lambda \phi_{1}+(1-\lambda) \phi_{2}}\right) \\
= & \left(\lambda \phi_{1}+(1-\lambda) \phi_{2}\right) \\
& \times f\left(\frac{\lambda \phi_{1}}{\lambda \phi_{1}+(1-\lambda) \phi_{2}} \frac{y_{1}}{\phi_{1}}+\frac{(1-\lambda) \phi_{2}}{\lambda \phi_{1}+(1-\lambda) \phi_{2}} \frac{y_{2}}{\phi_{2}}\right) \\
\leq & \lambda \phi_{1} f\left(\frac{y_{1}}{\phi_{1}}\right)+(1-\lambda) \phi_{2} f\left(\frac{y_{2}}{\phi_{2}}\right)
\end{aligned}
$$

for any $\left(y_{1}, \phi_{1}\right),\left(y_{2}, \phi_{2}\right) \in R^{n} \times R_{+}$, and $\lambda \in[0,1]$. Hence, the objective function of problem $\left(P^{\prime}\right)$ is convex in $(y, \phi)$.

Now, since $g$ is affine, which is also convex, $\phi g(y / \phi)$ can be proved to be convex, by following similar steps. Therefore, the feasible constraint set is a convex set and we conclude that problem $\left(P^{\prime}\right)$ is a convex program if $f$ is convex and $g$ is an affine function on $S$.

Henceforth, from the Charnes-Cooper transformation, we note that if the optimal solution $\left(y^{*}, \phi^{*}\right)$ of problem $\left(P^{\prime}\right)$ is found, then $x^{*}=y^{*} / \phi^{*}$ is optimal for problem $(P)$. 


$$
\left.U 5^{\prime}\right|_{\substack{P_{\mathrm{t}}=P_{1}^{*} \\ P_{\mathrm{norm}}=P_{\text {norm }, 1}}}=\frac{\frac{w_{1} \Psi_{\mathrm{EE}, 1}}{\epsilon} \mathrm{EC}-\left(w_{1} \Psi_{\mathrm{EE}, 1}\left(P_{\mathrm{c}}+\frac{1}{\epsilon} \bar{P}_{\mathrm{t}}\right)+\left(1-w_{1}\right) \Psi_{\mathrm{EC}, 1}\right) \mathrm{EC}^{\prime}}{\mathrm{EC}^{2}}=0,
$$

$$
U 5^{\prime} \mid \begin{aligned}
& \bar{P}_{\mathrm{t}}=P_{1}^{*} \\
& P_{\text {norm }}=P_{\text {norm }, 1}+\Delta P_{\text {norm }}
\end{aligned}=\frac{\Psi_{\mathrm{EE}, 2}\left[\frac{w_{1} \mathrm{EC}}{\epsilon}-\left(\frac{w_{1}}{\epsilon} P_{1}^{*}+P_{\mathrm{c}}+\frac{\left(1-w_{1}\right)}{\epsilon}\left(P_{\text {norm }, 1}+\Delta P_{\text {norm }}\right)\right) \mathrm{EC}^{\prime}\right]}{\mathrm{EC}^{2}},
$$

\section{APPENDIX E \\ PROOF OF LEMMA 3}

For a system with optimal average transmit power $P_{1}^{*}$, and normalization values $\Psi_{\mathrm{EE}, 1}=\left.\mathrm{EE}\right|_{P_{\mathrm{t}}=P_{\text {norm } 1}}$ and $\Psi_{\mathrm{EC}, 1}=$ $\left.\mathrm{EC}\right|_{\bar{P}_{\mathrm{t}}}=P_{\mathrm{norm}, 1}$, take the first derivative of the function $U 5$, which yields (25), as shown at the top of the page, and it simplifies to

$$
\frac{w_{1} \mathrm{EC}}{\epsilon}-\left(\frac{w_{1}}{\epsilon} P_{1}^{*}+P_{\mathrm{c}}+\frac{\left(1-w_{1}\right)}{\epsilon} P_{\mathrm{norm}, 1}\right) \mathrm{EC}^{\prime}=0 .
$$

Then, consider a system with a larger $P_{\text {norm }}$, i.e., $P_{\text {norm }, 2}=$ $P_{\text {norm }, 1}+\Delta P_{\text {norm }}, \Delta P_{\text {norm }}>0$. In this system, the optimal input power value at which the tradeoff formulation can be maximized is denoted by $P_{2}^{*}$, and normalization values are $\Psi_{\mathrm{EE}, 2}$ and $\Psi_{\mathrm{EC}, 2}$. Replacing $P_{\text {norm, } 1}$ in (25) with $P_{\text {norm, } 2 \text {, we }}$ have (27), as shown at the top of the page. By using (26), it reduces to

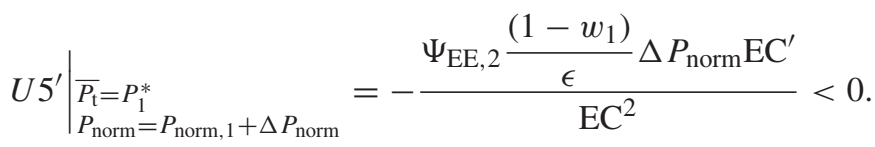

From Theorem 2, we know that $U 5$ strictly decreases with the average transmit power until reaching the minimum, then it becomes a monotonically increasing function. Therefore, (28) means that $U 5$ with a larger $P_{\text {norm }}$ decreases at $P_{1}^{*}$ and has not reached its minimum yet, which means $P_{2}^{*}$ must be bigger than $P_{1}^{*}$. It is easy to prove that the average optimal power monotonically decreases with $w_{1}$, which is omitted here. This completes the proof of Lemma 3.

\section{REFERENCES}

[1] A. J. Goldsmith, Wireless Communications. Cambridge, U.K.: Cambridge Univ. Press, 2005.

[2] ITU Statistics. (2014, May). The World in 2014: ICT Facts and Figures [Online]. Available: http://www.itu.int/en/ITU-D/Statistics/Pages/facts/ default.aspx

[3] GSMA Intelligence. (2014, Dec.). Understanding 5G: Perspectives on Future Technological Advancements in Mobile [Online]. Available: https://gsmaintelligence.com/research/2014/12/understanding-5g/451/

[4] C. Gunaratne et al., "Reducing the energy consumption of ethernet with adaptive link rate (ALR)," IEEE Trans. Comput., vol. 57, no. 4, pp. 448461, Apr. 2008.

[5] Smart2020, "Enabling the low-carbon economy in the information age," The Climate Group, London, U.K., Tech. Rep., 2008.

[6] W. D. Nordhaus, "To slow or not to slow: The economics of the greenhouse effect," Econ. J., vol. 101, no. 407, pp. 920-937, Jul. 1991.

[7] A. P. Bianzino et al., "A survey of green networking reasearch," IEEE Commun. Surveys Tuts., vol. 14, no. 1, pp. 3-20, Feb. 2012.

[8] C. W. Tan, D. P. Palomar, and M. Chiang, "Energy-robustness tradeoff in cellular network power control," IEEE/ACM Trans. Netw., vol. 17, no. 3, pp. 912-925, Jun. 2009
[9] L. Zhang and C. W. Tan, "Cognitive radio network duality and algorithms for utility maximization," IEEE J. Sel. Areas Commun., vol. 31, no. 3, pp. 500-513, Mar. 2013.

[10] Y. Li et al., "Energy-efficient subcarrier assignment and power allocation in OFDMA systems with max-min fairness guarantees," IEEE Trans. Commun., vol. 63, no. 9, pp. 3183-3195, Sep. 2015

[11] X. Zhai, L. Zheng, and C. W. Tan, "Energy-infeasibility tradeoff in cognitive radio networks: Price-driven spectrum access algorithms," IEEE J. Sel. Areas Commun., vol. 32, no. 3, pp. 528-538, Mar. 2014.

[12] C. Bae and W. E. Stark, "End-to-end energy-bandwidth tradeoff in multihop wireless networks," IEEE Trans. Inf. Theory, vol. 55, no. 9, pp. 4051-4066, Sep. 2009.

[13] C. Xiong et al., "Energy- and spectral-efficiency tradeoff in downlink OFDMA networks," IEEE Trans. Wireless Commun., vol. 10, no. 11, pp. 3874-3886, Nov. 2011.

[14] Y. Li et al., "Energy efficiency and spectral efficiency tradeoff in interference-limited wireless networks," IEEE Commun. Lett., vol. 17, no. 10, pp. 1924-1927, Oct. 2013.

[15] X. Chen and S. Ouyang, "Energy- and spectral-efficiency trade-off in OFDMA-based cooperative cognitive radio networks," Int. J. Distrib. Sensor Netw., Feb. 2014.

[16] C. He et al., "Energy- and spectral-efficiency tradeoff for distributed antenna systems with proportional fairness," IEEE J. Sel. Areas Commun., vol. 31, no. 5, pp. 894-902, May 2013.

[17] O. Amin et al., "Energy efficiency and spectral efficiency trade-off for OFDM systems with imperfect channel estimation," in Proc. IEEE Int. Conf. Commun. (ICC), Sydney, NSW, Australia, Jun. 2014, pp. $3553-$ 3558.

[18] D. Wu and R. Negi, "Effective capacity: A wireless link model for support of quality-of-service," IEEE Trans. Wireless Commun., vol. 2, no. 4, pp. 630-643, Jul. 2003.

[19] M. Gursoy, D. Qiao, and S. Velipasalar, "Analysis of energy efficiency in fading channels under QoS constraints," IEEE Trans. Wireless Commun., vol. 8, no. 8, pp. 4252-4263, Aug. 2009.

[20] L. Musavian and Q. Ni, "Delay-QoS-driven spectrum and energy efficiency tradeoff," in Proc. IEEE Int. Conf. Commun. (ICC), Sydney, NSW, Australia, Jun. 2014, pp. 4981-4986.

[21] L. Musavian and Q. Ni, "Effective capacity maximization with statistical delay and effective energy efficiency requirements," IEEE Trans. Wireless Commun., vol. 14, no. 7, pp. 3824-3835, Jul. 2015.

[22] W. Cheng, X. Zhang, and H. Zhang, "Joint spectrum and power efficiencies optimization for statistical QoS provisionings over SISO/MIMO wireless networks," IEEE J. Sel. Areas Commun., vol. 31, no. 5, pp. $903-$ 915, May 2013.

[23] X. Chen et al., "Tradeoff between energy efficiency and spectral efficiency in a delay constrained wireless system," Wireless Commun. Mobile Comput., vol. 15, pp. 1945-1956, Mar. 2014

[24] L. Musavian and T. Le-Ngoc, "Energy-efficient power allocation over Nakagmi-m fading channels under delay-outage constraints," IEEE Trans. Wireless Commun., vol. 13, no. 8, pp. 4081-4091, Aug. 2014.

[25] J. Tang and X. Zhang, "Quality-of-service driven power and rate adaptation over wireless links," IEEE Trans. Wireless Commun., vol. 6, no. 8, pp. 3058-3068, Aug. 2007.

[26] M. K. Simon and M.-S. Alouini, Digital Communication Over Fading Channels: A Unified Approach to Performance Analysis. Hoboken, NJ, USA: Wiley, 2000.

[27] M. Abramowitz and I. A. Stegun, Handbook of Mathematical Functions. New York, NY, USA: Dover, 1965.

[28] S. Ruzika and M. M. Wiecek, "Survey paper: Approximation methods in multiobjective programming," J. Optim. Theory Appl., vol. 126, no. 3, pp. 473-501, Sep. 2005.

[29] J. S. A. R. T. Marler, "Survey of multi-objective optimization methods for engineering," Struct. Multidiscip. Optim., vol. 26, no. 6, pp. 369-395, Apr. 2004. 
[30] L. Zhang and C. W. Tan, "Maximizing sum rates in cognitive radio networks: Convex relaxation and global optimization algorithms," IEEE J. Sel. Areas Commun., vol. 32, no. 3, pp. 667-680, Mar. 2014.

[31] J. A. Bucklew, Introduction to Rare Event Simulation. Berlin, Germany: Springer-Verlag, 2004

[32] C. S. Chang, "Stability, queue length, and delay of deterministic and stochastic queueing networks," IEEE Trans. Autom. Control, vol. 39, no. 5, pp. 913-931, May 1994

[33] A. Dembo and O. Zeitouni, Large Deviations Techniques and Applications, 2nd ed. Berlin, Germany: Springer-Verlag, 1998.

[34] A. Zappone and E. Jorswieck, "Energy efficiency in wireless networks via fractional programming theory, in Found. Trends Commun. Inf. Theory. Hanover, MA, USA: Now Publishers Inc., Jun. 2015, vol. 11.

[35] A. J. Goldsmith and P. P. Varaiya, "Capacity of fading channels with channel side information," IEEE Trans. Inf. Theory, vol. 43, no. 6, pp. 1986-1992, Nov. 1997.

[36] S. Schaible, "Parameter-free convex equivalent and dual programs of fractional programming problems," Zeitschrift Oper. Res., vol. 18, no. 5, pp. 187-196, Oct. 1974

[37] S. Schaible, "Fractional programming," Zeitschrift Oper. Res., vol. 27, no. 1, pp. 39-54, Dec. 1983

[38] M. Ehrgott, Multicriteria Optimization. New York, NY, USA: Springer, 2005.

[39] S. Boyd and L. Vandenberghe, Convex Optimization. Cambridge, U.K.: Cambridge Univ. Press, 2004.

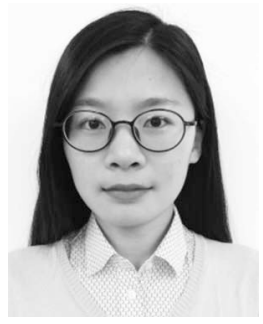

Wenjuan Yu received her B.Sc. degree in Electronic and Information Engineering, from Shandong University of Technology, China, in 2010 and her M.Sc. degree from the School of Telecommunications Engineering, Xidian University, China, in 2013. She is currently working toward the Ph.D. degree with the School of Computing and Communications, InfoLab21, Lancaster University, Lancaster, UK. Her research interests include resource allocation, cross-layer optimization toward green communications, delay QoS provisioning.

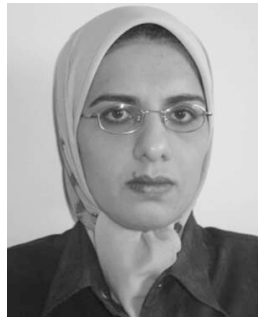

Leila Musavian (S'05-M'07) received the Ph.D. degree in telecommunications from Kings College London, London, U.K., in 2006. She is currently working as a Lecturer in communications with the School of Computing and Communications, InfoLab21, Lancaster University, Lancaster, U.K. Prior to that (2010-2012), she was a Research Associate with McGill University. She was a Postdoctoral Fellow at INRS-EMT, University of Quebec, Canada, from 2006 to 2008. Her research interests include radio resource management for next generation wireless networks, CRNs, green communication, energy-efficient transmission techniques, cross-layer design for delay QoS provisioning and 5G systems. She is an Editor of IEEE TRANSACTIONS ON Wireless COMMUNICATIONS.

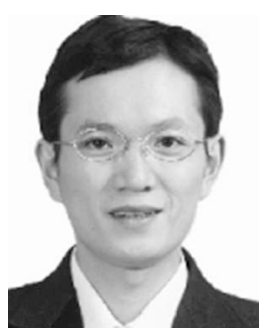

Qiang Ni (M'04-SM'08) received the B.Sc., M.Sc., and $\mathrm{Ph} . \mathrm{D}$. degrees from Huazhong University of Science and Technology, China, all in engineering. $\mathrm{He}$ is a Professor and the Head of Communication Systems Group, School of Computing and Communications, Lancaster University, InfoLab21, Lancaster, U.K. Previously, he led the Intelligent Wireless Communication Networking Group, Brunel University London, Middlesex, U.K. His research interests include future generation communications and networking, including green communications and networking, cognitive radio network systems, heterogeneous networks, small cell and ultra dense networks, 5G, SDN, energy harvesting, wireless information and power transfer, IoTs and vehicular networks in which areas he had already published over 120 papers. He was an IEEE 802.11 Wireless Standard Working Group Voting member and a Contributor to the IEEE WIRELESS STANDARDS. 